\title{
Large Eddy Simulation of Turbulent Spray Combustion
}

\author{
Abolfazl Irannejad*, Araz Banaeizadeh ${ }^{1}$, Farhad Jaberi \\ Department of Mechanical Engineering, Michigan State University East Lansing, MI 48824
}

\begin{abstract}
The two-phase filtered mass density function (FMDF) method is employed for large eddy simulation (LES) of high speed evaporating and combusting nheptane sprays using simple (global) and complex (skeletal) chemical kinetic mechanisms. The resolved fluid velocity and pressure fields are obtained by solving the filtered compressible Navier-Stokes equations with high-order Eulerian finite difference methods. The liquid spray and gas scalar (temperature and species mass fractions) fields are both obtained by Lagrangian stochastic models. The chemistry calculation is accelerated by incorporating the parallel in situ adaptive tabulation (ISAT) method. There are two-way interactions among Eulerian and Lagrangian fields. Simulations of evaporating sprays with and without combustion indicate that the two-phase LES/FMDF results are consistent and compare well with the available experimental data at different gas temperatures and oxygen concentrations. The spray controlled flame tends to move away from a diffusion flame structure toward a premixed one as the oxygen concentration decreases and/or the ambient gas temperature increases because of changes in spray-induced turbulence and mixing. The LES/FMDF results for ignition delay show more sensitivity to the chemical kinetic model at lower gas temperatures due to slower reaction and stronger turbulence-chemistry interactions. The liftoff length is less sensitive to the kinetics.
\end{abstract}

Keywords: Turbulent Combustion, Spray Flame, Large-Eddy Simulation,

\footnotetext{
* Corresponding author

Email address: iranneja@msu.edu (Abolfazl Irannejad)

${ }^{1}$ Current address: Altair Engineering, Mountain View, CA 94043
} 


\section{Introduction}

Numerical simulation of turbulent spray combustion is extremely challenging due to multi-scale and multi-time nature of liquid-gas interactions and the complexity of finite-rate evaporation, mixing and multi-step reactions $[1,2,3]$. Direct numerical simulation (DNS) of high speed reacting sprays with complex chemistry models is not currently feasible; the latest published DNS work in this area is limited to relatively simple dilute two-phase homogeneous turbulent reacting flows [4]. Large-eddy simulation (LES) models, on the other hand are expected to be applicable to turbulent spray combustion. LES has been widely applied to single-phase reacting flows [5, 6, 7]. However, its use for turbulent spray combustion simulations has been somewhat limited mainly because of difficulties in modeling of subgrid-scale (SGS) interactions among droplets, turbulence and combustion and the computational cost associated with complex chemical kinetic mechanisms [8]. Recently, different computational methods have been suggested for the simulations of spray-turbulence-combustion interactions $[9,10,11,12]$. These simulations, which often involved complicated geometries, have been conducted with different fuels and reaction models. However, the incorporated chemical kinetic mechanisms were relatively simple. The ignition and flame stabilization show strong dependence on the chemical kinetics in spray systems[13], making the choice of reaction model also important.

The traditional approach to the spray simulation is to compute the gas-phase by a grid-based Eulerian method and the spray by a particle-based Lagrangian method. In this hybrid approach, submodels are required to account for the various physical processes taking place at small time and length scales in both Eulerian and Lagrangian fields. Provided that the SGS particle motion and scalar fluxes are correctly modeled, the accuracy of simulations is still dependent on the models used for the liquid evaporation, micro-mixing, chemical kinetics and turbulence-combustion interactions $[14,8]$. Transported probabil- 
ity density function (PDF) methods have proven to be particularly effective in accounting for turbulence-chemistry interactions [7]. Filtered mass density function (FMDF) is a single-point SGS PDF model for LES of turbulent reacting flows [15]. In this approach, the joint statistics of turbulent variables at subgrid level are obtained by solving the transport equation for the FMDF. The main advantage of FMDF is that the single-point statistics like reaction terms appear in a closed form in its formulation. This allows simulations of various types of reactions (slow, fast, premixed, non-premixed, etc.) with different chemical kinetic models $[6,16,17,18,19,20,21]$. Li et al. [22] developed a two-phase FMDF model for simulation of two-phase reacting flows. They validated the model by comparison with DNS data in a spatially developing droplet-laden reactive mixing layer.

Depending on the type of spray and flame, the reaction time scales can be very different or comparable to those of the turbulence and the spray. This has led to the development and usage of reaction mechanisms at various levels of details and comprehensiveness [23]. For some of the hydrocarbon fuels such as n-heptane [24], complex (skeletal or reduced) kinetics models are available beyond simple global mechanisms [25, 26, 27]. Complex reaction models are normally needed for high speed spray combustion simulations. However, the computational cost of making the chemistry calculations with complex kinetics is often prohibitive and efficient implementation of chemistry is necessary.

Several different methods have been proposed for efficient implementation of chemical reaction in computational models such as LES/FMDF. Among them are the storage/retrieval algorithms [28] including the structured look-up tabulation [29], the repro-modeling [30], the artificial neural networks (ANN) [31]-[32], the in situ adaptive tabulation (ISAT) [33]-[34], the piecewise reusable implementation of solution mapping (PRISM) [35], and the high dimension model representations (HDMR) [36]. The in situ adaptive tabulation or ISAT, is a storage and retrieval methodology which has been widely adopted especially with PDF methods. ISAT algorithm has been applied to statistically stationary flows such as non-premixed piloted jet flames [37] with a speed-up factor 
of 100-1000. However, it has also been applied to the calculation of transient processes in internal combustion (IC) engines [38] with a speed up factor of 10. The original ISAT algorithm by Pope [33] has been improved and used for parallel and efficient PDF calculations [34, 39, 40].

For evaluation of turbulent spray combustion models under engine relevant thermochemical conditions, a reliable experimental dataset is required. The Engine Combustion Network (ECN) [41] provides an open forum for experimental and computational studies and collaborations on turbulent spray combustion. One of the configurations considered by ECN is a constant volume turbulent spray combustion chamber that has the thermochemical conditions that are representative of those in modern direct injection (DI) compression ignition engines $[42,43,44]$. In the DI systems, the spray is injected with a very high velocity into a low speed, high temperature ambient gas and therefore is a significant source of generating the gas flow turbulence. As the liquid fuel has to go through breakup, droplet formation, and evaporation before reaction, all parts of the spray evolution is important to the combustion. The rate of evaporation, alone is dependent on details of the breakup and the droplet size distribution but is also affected by the local gas temperature and the vapor concentration which are dependent on the spray induced turbulent flow and turbulent mixing $[42,45,46]$. Most of the numerical studies of sprays reported by ECN have been based on unsteady RANS with different turbulent-combustion models [47, 48, 49]. Very recently, the transported PDF method has been used for unsteady spray combustion simulations $[13,50]$.

In this paper, the LES and spray models are coupled with the two-phase FMDF for simulations of high speed evaporating and reacting sprays with complex chemical kinetic mechanisms. The goals are to first establish the reliability and accuracy of the LES/FMDF methodology for turbulent spray combustion simulations and then use the model for detailed study of spray-turbulencecombustion interactions in several high speed sprays. The numerical data provide a wealth of information on these interactions for very different spray and gas flow conditions. Details of the two-phase FMDF methodology and combus- 
tion chemistry calculations are described in the next section, followed by the results obtained with the LES/FMDF code.

\section{Mathematical and Computational Model}

The LES/FMDF/Spray model has three main mathematical/computational components: (1) the filtered gas dynamic equations, (2) the FMDF equations and (3) the Lagrangian spray equations. These are presented in the following sections, together with a brief description of the numerical solution methods. The developed computational method for the solution of two-phase LES/FMDF has one Eulerian and two Lagrangian solvers. The gas phase is treated by a hybrid methodology. The compressible LES equations for velocity and pressure are solved by high-order compact finite difference methods via a grid-based Eulerian multiblock flow solver $[16,51,52]$. The scalar field (i.e. the species mass fractions and enthalpy) is computed by the FMDF. The FMDF is obtained by solution of its transport equation with an efficient Lagrangian stochastic Monte Carlo method, with spray effects included [15, 22]. The spray is simulated with a non-equilibrium Lagrangian particle method together with a stochastic droplet breakup model which allows full two-way mass, momentum, and energy coupling between phases [51]. The chemistry is based on finite rate skeletal kinetics and is implemented by ISAT and chemistry load balancing for parallel processing. 


\subsection{Filtered Gas Dynamic Equations}

The gas phase is simulated by solving the following compressible Favrefiltered continuity, momentum, energy and species mass fraction equations.

$$
\begin{gathered}
\frac{\partial\langle\rho\rangle_{l}}{\partial t}+\frac{\partial\langle\rho\rangle_{l}\left\langle u_{i}\right\rangle_{L}}{\partial x_{i}}=\left\langle\dot{S}_{M a s s}^{s p}\right\rangle_{l}, \\
\frac{\partial\langle\rho\rangle_{l}\left\langle u_{i}\right\rangle_{L}}{\partial t}+\frac{\partial\langle\rho\rangle_{l}\left\langle u_{i}\right\rangle_{L}\left\langle u_{j}\right\rangle_{L}}{\partial x_{j}}=-\frac{\partial\langle p\rangle_{l}}{\partial x_{i}}+\frac{\partial\left\langle\tau_{i j}\right\rangle_{l}}{\partial x_{j}}-\frac{\partial\left(\tau_{i j}^{s g s}\right)}{\partial x_{j}}+\left\langle\dot{S}_{m i}^{s p}\right\rangle_{l}, \\
\frac{\partial\langle\rho\rangle_{l}\langle E\rangle_{L}}{\partial t}+\frac{\partial\left(\langle\rho\rangle_{l}\langle E\rangle_{L}+\langle p\rangle_{l}\right)\left\langle u_{i}\right\rangle_{L}}{\partial x_{i}}=-\frac{\partial\left(\left\langle q_{i}\right\rangle_{l}+H_{i}^{s g s}\right)}{\partial x_{i}}+\langle\dot{Q}\rangle_{l}+\left\langle\dot{S}_{E}^{s p}\right\rangle_{l} \\
+\frac{\partial\left[\left(\left\langle\tau_{i j}\right\rangle_{l}-\tau_{i j}^{s g s}\right)\left\langle u_{j}\right\rangle_{L}\right]}{\partial x_{i}}, \\
\frac{\partial\langle\rho\rangle_{l}\left\langle\phi_{\alpha}\right\rangle_{L}}{\partial t}+\frac{\partial\langle\rho\rangle_{l}\left\langle u_{i}\right\rangle_{L}\left\langle\phi_{\alpha}\right\rangle_{L}}{\partial x_{i}}=-\frac{\partial\left(\left\langle J_{i}^{\alpha}\right\rangle_{l}+J_{i}^{\alpha s g s}\right)}{\partial x_{i}}+\left\langle\rho \dot{S}_{\alpha}\right\rangle_{l}+\left\langle\dot{S}_{\alpha}^{s p}\right\rangle_{l} .
\end{gathered}
$$

In Equations 1 to $4,\langle\rangle_{l}$ and \langle\rangle$_{L}$ represent the filtering and Favre-filtering operations, respectively. The primary variables are filtered density $\langle\rho\rangle_{l}$, the Favrefiltered velocity $\left\langle u_{i}\right\rangle_{L}$, the Favre-filtered total energy $\langle E\rangle_{L}=\langle e\rangle_{L}+\frac{1}{2}\left\langle u_{i}\right\rangle_{L}\left\langle u_{i}\right\rangle_{L}$ and the Favre-filtered scalar mass fraction $\left\langle\phi_{\alpha}\right\rangle_{L}$. The filtered viscous stress tensor, $\left\langle\tau_{i j}\right\rangle_{l}$, is assumed to be a linear function of the Favre-filtered strain rate, $\left\langle S_{i j}\right\rangle_{L}$, for the simulated Newtonian fluid, and the filtered heat flux vector, $\left\langle q_{i}\right\rangle_{l}$ and species diffusion, $\left\langle J_{i}^{\alpha}\right\rangle$, are evaluated based on Fourier and Fick's laws. The specific internal energy of the mixture is defined as:

$$
\langle e\rangle_{L}=\sum_{\alpha=1}^{N_{s}}\left\langle\phi_{\alpha}\right\rangle_{L} h_{\alpha}\left(\langle T\rangle_{L}\right)-\left\langle R_{m i x}\right\rangle_{L}\langle T\rangle_{L},
$$

where the species sensible enthalpy is

$$
h_{\alpha}(T)=\int_{T_{r e f}}^{T} c_{p, \alpha} d T .
$$

The mixture gas constant is defined based on the universal gas constant $R^{0}$, the species molecular weight $M W_{\alpha}$ and the species mass fractions as

$$
\left\langle R_{m i x}\right\rangle_{L}=R^{0} \sum_{\alpha=1}^{N_{s}} \frac{\left\langle\phi_{\alpha}\right\rangle_{L}}{M W_{\alpha}} .
$$

The filtered form of ideal gas equation of state is used to close the system of equations. The unclosed subgrid scale terms which appear in the filtered 
equations are closed by gradient type closures $[51,53,54]$. In these closures, the characteristic length and velocity for defining the eddy viscosity are provided by the local grid size $\bar{\Delta}$ and the subgrid kinetic energy, $k_{s g s}$ as $\nu_{t}=c_{\nu} \bar{\Delta} \sqrt{ } k_{s g s}$. The subgrid kinetic energy is obtained by the solution of its transport equation, which includes spray mass and momentum coupling terms [51].

\subsection{Two-Phase Filtered Mass Density Function}

The scalar FMDF is a joint probability density function of the scalars (species mass fractions and energy) at the subgrid-level, which was originally developed by Jaberi et al. [15] for single-phase reacting flows and has proven to be very reliable and accurate for these flows $[16,17,18,19,21,55,56,57]$. Li et al. [22] extended the original FMDF to two-phase flows. Banaeizadeh et al. [52] included the compressibility effects in the scalar FMDF methodology.

The definition for FMDF is,

$$
F_{L}(\mathbf{\Psi} ; \mathbf{x}, t)=\int_{-\infty}^{+\infty} \rho\left(\mathbf{x}^{\prime}, t\right) \xi\left(\Psi, \Phi\left(\mathbf{x}^{\prime}, t\right)\right) G\left(\mathbf{x}^{\prime}-\mathbf{x}\right) d \mathbf{x}^{\prime}
$$

where $G$ denotes the filter function, $\boldsymbol{\Psi}$ is the scalar vector in the sample space and $\xi$ is the fine grained density [58], defined based on a series of delta functions. The scalar vector includes species mass fractions $\left(\alpha=1, N_{s}\right)$ and specific sensible enthalpy $\left(\alpha=N_{s}+1\right)$.

The FMDF transport equation is obtained by inserting the instantaneous unfiltered scalar equation into the time derivative of the fine grained density and filtering that. For a compressible two-phase reacting system with small liquid volume fraction, the FMDF equation can be written in the following 
form:

$$
\begin{aligned}
\frac{\partial F_{L}}{\partial t}+\frac{\partial}{\partial x_{i}}\left[\left\langle u_{i}(\mathbf{x}, t)\right\rangle_{L} F_{L}\right] & \left.=-\frac{\partial}{\partial x_{i}}\left[\left\langle u_{i}(\mathbf{x}, t) \mid \mathbf{\Psi}\right\rangle_{l}-\left\langle u_{i}\right\rangle_{L}\right) F_{L}\right] \\
& +\frac{\partial}{\partial \psi_{\alpha}}\left[\left\langle\frac{1}{\rho(\boldsymbol{\Phi})} \frac{\partial J_{i}^{\alpha}}{\partial x_{i}} \mid \boldsymbol{\Psi}\right\rangle_{l} F_{L}\right] \\
& -\frac{\partial}{\partial \psi_{\alpha}}\left[\dot{S}_{\alpha}(\mathbf{\Psi}) F_{L}\right)-\frac{\partial}{\partial \psi_{\alpha}}\left[\left\langle\dot{S}_{\alpha}^{\text {comp }} \mid \mathbf{\Psi}\right\rangle_{l} F_{L}\right] \\
& -\frac{\partial}{\partial \psi_{\alpha}}\left[\frac{\dot{S}_{\alpha}^{s p}|\boldsymbol{\Psi}\rangle F_{L}}{\rho(\boldsymbol{\Psi})}\right]+\frac{\partial}{\partial \psi_{\alpha}}\left[\frac{\left\langle\dot{S}_{\text {Mass }}^{s p} \mid \boldsymbol{\Psi}\right\rangle \psi_{\alpha} F_{L}}{\rho(\boldsymbol{\Psi})}\right] \\
& +\frac{\left\langle\dot{S}_{\text {Mass }}^{s p} \mid \boldsymbol{\Psi}\right\rangle F_{L}}{\rho(\boldsymbol{\Psi})} .
\end{aligned}
$$

It is noted here that in the above FMDF equation reaction terms are closed but in conventional LES methods they are not closed and have to be modeled. The first term in the right hand side (RHS) of Equation 9 represents the SGS convection which is modeled with a gradient type closure:

$$
\left(\left\langle u_{i}(\mathbf{x}, t) \mid \Psi\right\rangle_{l}-\left\langle u_{i}\right\rangle_{L}\right) F_{L}=-\Gamma_{t} \frac{\partial\left(F_{L} /\langle\rho\rangle_{l}\right)}{\partial x_{i}},
$$

where $\Gamma_{t}=\mu_{t} / P r_{t}$ or $\Gamma_{t}=\mu_{t} / S c_{t}$ is the turbulent diffusivity. The term due to molecular diffusion is decomposed into two parts, the molecular transport and the SGS dissipation. The SGS dissipation is modeled with the linear meansquare estimation (LMSE) [59]-[60] or the interaction by exchange with the mean (IEM) model [61]:

$$
\frac{\partial}{\partial \psi_{\alpha}}\left[\left\langle\frac{1}{\rho(\boldsymbol{\Phi})} \frac{\partial J_{i}^{\alpha}}{\partial x_{i}} \mid \boldsymbol{\Psi}\right\rangle_{l} F_{L}\right]=\frac{\partial}{\partial x_{i}}\left[\Gamma \frac{\partial}{\partial x_{i}}\left(\frac{F_{L}}{\langle\rho\rangle_{l}}\right)\right]+\frac{\partial}{\partial \psi_{\alpha}}\left[\Omega_{m}\left(\psi_{\alpha}-\left\langle\phi_{\alpha}\right\rangle_{L}\right) F_{L}\right],
$$

where the molecular diffusivity is $\Gamma=\mu / \operatorname{Pr}$ or $\Gamma=\mu / S c$ and the SGS mixing frequency is $\Omega_{m}=\frac{1}{2} c_{\phi}\left(\Gamma+\Gamma_{t}\right) /\left(\bar{\Delta}^{2}\langle\rho\rangle_{l}\right)$.

The compressible source term due to pressure in the scalar FMDF is obtained by taking into account the total derivative of filtered pressure for the mixture enthalpy as [52]:

$$
\left\langle\dot{S}_{\alpha}^{c o m p} \mid \Psi\right\rangle_{l}=\frac{1}{\langle\rho\rangle_{l}}\left(\frac{\partial\langle p\rangle_{l}}{\partial t}+\left\langle u_{i}\right\rangle_{L} \frac{\partial\langle p\rangle_{l}}{\partial x_{i}}\right), \alpha=N_{s}+1 .
$$

Due to low Mach number flow in the simulated spray flames, the viscous dissipation term in enthalpy equation is small and neglected. 
The last three terms on the RHS of Equation 9 represent the spray/droplet effects on FMDF. They involve particle source/sink terms affecting the gas composition and density. The spray source term due to added mass to the gas is approximated as

$$
\frac{\left\langle\dot{S}_{\text {Mass }}^{s p} \mid \Psi\right\rangle F_{L}}{\rho(\Psi)}=\frac{\left\langle\dot{S}_{\text {Mass }}^{s p}\right\rangle_{l} F_{L}}{\langle\rho\rangle} .
$$

The spray source term to the scalar field (fuel mass fraction and enthalpy) is approximated as

$$
\frac{\left\langle\dot{S}_{\alpha}^{s p} \mid \Psi\right\rangle F_{L}}{\rho(\boldsymbol{\Psi})}=\frac{\left\langle\dot{S}_{\alpha}^{s p}\right\rangle_{l} F_{L}}{\langle\rho\rangle} .
$$

The term due to interaction of the scalar field with the spray added mass is approximated as

$$
\frac{\left\langle\psi_{\alpha} \dot{S}_{M a s s}^{s p} \mid \Psi\right\rangle F_{L}}{\rho(\Psi)}=\frac{\psi_{\alpha}\left\langle\dot{S}_{M a s s}^{s p}\right\rangle_{l} F_{L}}{\langle\rho\rangle} .
$$

The modeled FMDF transport equation is closed and may be solved to get the scalar FMDF and consequently all single-point statistical information concerning reactive species and temperature at various times and locations, including the first moments or filtered variables. Also, by integrating the FMDF equation in $\mathrm{PDF}$ or composition space, one can recover the transport equations for the first, second and all the other SGS scalar moments. This indicates a mathematical consistency between the FMDF and the conventional LES methods which directly solve the moment equations. The consistency for reacting cases can only be established by calculating the reaction terms from the FMDF and use it in the conventional LES method.

The most convenient means of solving the FMDF transport equation is via the Lagrangian Monte Carlo (MC) procedure [62]. With the Lagrangian procedure, the FMDF is represented by an ensemble of computational "stochastic elements" or "Monte Carlo particles". These notional particles evolve through a "stochastic process", described by a set of stochastic differential equations (SDEs) $[63,64]$. The stochastic process is described by the Fokker-Planck equation. A comparison between the Fokker-Planck equation and the FMDF equation under consideration identifies the parameters of the stochastic equations [15]. A unique feature of this stochastic procedure is that the large-scale, 
subgrid-scale, and molecular mixing, the chemical reaction and the two-phase effects can be separately incorporated and evaluated, offering a systematic way to compute and interpret these processes in the LES/FMDF calculations. The simplest means of simulating the spatial transport in this equation is via the Euler-Maruyamma approximation [63]. Higher order numerical schemes are available, but one must be cautious in using them for LES since the diffusion term in stochastic equation depends on the stochastic process. The numerical scheme must preserve the Ito-Gikhman nature of the process. The EulerMaruyamma approximation provides sufficient accuracy for the flows studied here.

In the stochastic Lagrangian method, each MC particle is transported in the "physical space" by the combined actions of large scale convection and diffusion (molecular and subgrid):

$$
d X_{i}^{+}=\left[\left\langle u_{i}\right\rangle_{L}+\frac{1}{\langle\rho\rangle_{l}} \frac{\partial\left(\Gamma+\Gamma_{t}\right)}{\partial x_{i}}\right] d t+\sqrt{\frac{2\left(\Gamma+\Gamma_{t}\right)}{\langle\rho\rangle_{l}}} d W_{i},
$$

where $d W_{i}$ is the increment of the Wiener process [63]. In addition, transport in the "composition space" occurs due to SGS/molecular mixing, chemical reaction, pressure variation and droplet heat and mass transfer.

$d \phi_{\alpha}^{+}=-\Omega_{m}\left(\phi_{\alpha}^{+}-\left\langle\phi_{\alpha}\right\rangle_{L}\right)+\dot{S}_{\alpha}\left(\phi^{+}\right) d t+\left\langle\dot{S}_{\alpha}^{c o m p}\right\rangle_{l} d t+\left[\frac{\left\langle\dot{S}_{\alpha}^{s p}\right\rangle_{l}-\phi_{\alpha}^{+}\left\langle\dot{S}_{M a s s}^{s p}\right\rangle_{l}}{\langle\rho\rangle_{l}}\right] d t$.

The spray terms in the FMDF equation are weighted averaged from FD grid points to the MC particle locations. To manage the number of MC particles and to reduce the computational cost, a procedure involving the use of non-uniform weights is considered. The variable weighting for particles allows the particle number density to stay above a certain minimum value regardless of density variations. It has been shown that the sum of weights within the ensemble averaging domain is related to the filtered fluid density as

$$
\langle\rho\rangle_{l} \approx \frac{\Delta m}{\Delta V} \sum_{n \in \Delta V} w^{(n)},
$$

where $\Delta V$ is the volume of the domain and $\Delta m$ is the mass of a MC particle with unit weight. For the spray simulation, particle weights should be modified 
due to added mass to the carrier gas from the evaporating droplets. The MC particle weight then is adjusted as

$$
d w^{(n)}=\frac{\Delta V}{\Delta m}\left\langle\dot{S}_{\text {Mass }}^{s p}\right\rangle_{l} d t .
$$

Here, it is assumed that the mean value of the evaporated mass is uniformly distributed over MC particles in each cell. Details of SGS mass transfer between droplets and Monte Carlo particles do not seem to have a very significant effect on the global spray and flame behavior simulated by LES/FMDF. However, in some circumstances, when for example the individual droplet combustion becomes important, it would be necessary to use a more detailed description of the mass transfer between the liquid and gas. This issue has been discussed in details in the context of RANS/PDF spray simulations in Ref. [8].

The effects of spray heat and mass transfer on the MC particles are implemented by a series of source terms in the particle equations. The modification of particle weights due to added mass to the gas is explained here. Other spray source terms are implemented in a similar manner. For a given number of Lagrangian MC particles at a given time, the carrier mass, $m$ can be written as:

$$
m=\int \rho(\mathbf{x}, t) d \mathbf{x}=\sum_{n} w^{(n)} \Delta m .
$$

From an Eulerian point of view, the mass conservation may be written as:

$$
\frac{d m}{d t}=\int \dot{S}_{M a s s}^{s p} d \mathbf{x} .
$$

From a Lagrangian point of view, the mass conservation can be described via Monte Carlo particles as:

$$
\frac{d m}{d t}=\Delta m \frac{d}{d t} \sum_{n} w^{(n)}=\Delta V_{E}\left\langle\dot{S}_{\text {Mass }}^{s p}\right\rangle_{l} .
$$

The mass conservation In the Lagrangian Monte Carlo procedure is then implemented by the following equations:

$$
\begin{aligned}
\frac{d w^{(n)}}{d t} & =\frac{\Delta V_{E}}{\Delta m}\left\langle\dot{S}_{\text {Mass }}^{s p}\right\rangle_{l} \\
\left\langle\dot{S}_{\text {Mass }}^{s p}\right\rangle_{l}^{+} & =\frac{w^{(n)}}{\sum_{n} w^{(n)}}\left\langle\dot{S}_{\text {Mass }}^{s p}\right\rangle_{l} .
\end{aligned}
$$


In the present hybrid methodology, the filtered temperature and species mass fractions may be calculated from both FD grid points and MC particles. This provides a unique opportunity for the assessment of LES-FD and FMDF-MC parts of the LES/FMDF solver. Mathematically, LES-FD and FMDF-MC results should be identical. Consistency of MC and FD data implies the numerical accuracy of both. As mentioned before, for establishing the consistency between FD and MC methods in reacting flows, the chemical source terms in the FD equations which are closed in the FMDF formulation are calculated from the MC particles. This is only possible in the LES/FMDF model. Apart from the temperature field which is required to be calculated in both LES-FD and FMDF-MC for numerical stability of compressible LES solver, only filtered fuel mass fraction equation is solved on finite difference grid points. This is done for showing the consistency of species and is not needed. The other species are obtained from FMDF and its MC particles.

\subsection{Combustion Chemistry and in situ Adaptive Tabulation}

As mentioned before, the chemical kinetics model plays a crucial role in LES of reacting spray and is important to the flame ignition, liftoff and extinction. In this work, the skeletal mechanism of Liu et al. [26] is used (unless otherwise mentioned) which has been extensively tested for high pressure non-premixed flames, conditions similar to those considered in our spray combustion simulations. This mechanism has 44 species and 185 reactions, counting forward and backward reactions individually. The modification of the scalar field due to reaction is carried out on MC particles with a fractional step method according to the following equation.

$$
d \phi_{\alpha}^{+}=\dot{S}_{\alpha}\left(\phi^{+}\right) d t, \alpha=1, N_{s}+1 .
$$

ISAT uses the ordinary differential equation (ODE) solver DDASAC [65] to integrate Equation 25 and stores the relevant information in a binary tree, with each termination node (or leaf) representing a record of the tabulation point for a specific composition, the reaction mapping in time step $\Delta t$ and the mapping 
gradient matrix. Using this matrix, for a given query composition close to a tabulated composition point, a linear approximation to the mapping is obtained under a controlled tolerance. An ellipsoid of accuracy (EOA) is used to approximate the region of accuracy. An EOA is a hyperellipsoid in composition space, representing the connected region in composition space containing a tree leaf. For a given query, ISAT traverses the tree until a leaf representing a point close to the query is reached. The following steps are taken to obtain an approximation to the corresponding reaction mapping [33, 34, 39]: (1) Retrieve: if the query falls within the ellipsoid of accuracy of the leaf, a linear approximation is returned. (2) Grow: otherwise, a function evaluation is performed to determine the reaction mapping. The error in linear approximation at the leaf is calculated and if the error is within the tolerance, the EOA of the leaf node is grown to include the query point. (3) Add: if the computed error is greater than the tolerance and the table is not full, then a new leaf is added. The error tolerance for ISAT is set to $1.0 \times 10^{-5}$. This value was shown to yield converged results for similar reacting spray simulations [13].

In large scale parallel LES/FMDF computations, the chemistry workload has to be distributed among the cores to reduce the overall computational time. Lu et al. [39] and Hiremath et al. [40] developed different distribution strategies for ISAT to balance the combustion chemistry workload on different computational cores. In the parallel calculations, each processor maintains its own ISAT table. However, during the reaction calculation, particles on one processor may be distributed to other processors. Among the distribution strategies tested by Hiremath et al. [40], the so called partitioned uniform random distribution (PURAN) has the highest efficiency and scalability which is adopted in this work. This strategy works in two stages: in stage 1 , all particles on a computational core are resolved chemically using the local ISAT table; then in stage 2, all parallel processors are partitioned into smaller groups and within each partition, the URAN strategy is used to uniformly distribute the chemistry workload. The URAN strategy aims at achieving statistically ideal load balancing by evenly distributing the chemistry workload among various processors. 


\subsection{Spray Equations}

The spray is modeled here with a Lagrangian model using blobs, drops and droplets [51]. The drop/droplet motion is simulated using Basset-BousinesqOseen (BBO) equation $[66,67,68]$. Under the assumptions of high liquid to gas density ratios, the Lagrangian equations describing the drop/droplet motion become

$$
\begin{gathered}
\frac{d \mathbf{x}_{p}}{d t}=\mathbf{u}_{p}, \\
\frac{d \mathbf{u}_{p}}{d t}=\frac{\mathbf{u}_{r e l}}{\tau_{p}} .
\end{gathered}
$$

In Equation 27, the relative velocity, $\mathbf{u}_{r e l}=\mathbf{u}_{g}-\mathbf{u}_{p}$, is calculated based on the gas velocity at the droplet location, $\mathbf{u}_{g}=\langle\mathbf{u}\rangle_{L}+\mathbf{u}^{*}$, which is reconstructed using the filtered velocity and the stochastic subgrid velocity $\mathbf{u}^{*}$ [69]. The stochastic subgrid velocity is calculated from the SGS velocity, $u_{s g s}=\sqrt{2 k_{s g s} / 3}$ and characteristic time scale $\tau_{L}^{*}$ :

$$
d u_{i}^{*}=-\frac{u_{i}^{*}}{\tau_{L}^{*}} d t+\sqrt{\frac{2 u_{s g s}^{2}}{\tau_{L}^{*}}} d W_{i},
$$

where $d W_{i}$ is an increment of the Wiener process [63]. The SGS velocity reconstruction is intended to cover a wide range of droplet Stokes numbers [69]. Furthermore, the droplet relative velocity is reduced by wake interactions of nearby droplets in the spray [70]. This modified relative velocity is then used for droplet motion, heat and mass transfer calculations. The particle response time is defined as

$$
\tau_{p}=\tau_{s t} / f_{d}\left(R e_{s l}\right),
$$

in which $\tau_{s t}=d_{p}^{2} \rho_{l} / 18 \mu_{g}$ is the droplet Stokes time scale and $f_{d}\left(R e_{s l}\right)$, the drag factor, is the finite Reynolds number $\left(R e_{s l}=\rho_{g} d_{p} u_{r e l} / \mu_{g}\right)$ correction to the Stokes Drag. The Reynolds number here is defined based on the droplet diameter, $d_{p}$. The drag factor $f_{d}$ includes the effects of evaporation through a blowing Reynolds number, $R e_{b w}$ for low Reynolds numbers $\left(R e_{s l}<100\right)[71]$. However for higher Reynolds numbers $\left(100<R e_{s l}<10^{5}\right)$, the evaporation effect on drag is neglected and standard drag factor for a sphere is used [66]. 
The drag factor is modified for a distorting drop, using the simple assumption that the drag coefficient of a distorting drop lies between the lower limit of a rigid sphere and the upper limit of a disk where the distortion is computed using the TAB model [72].

For high injection pressure evaporating and reacting sprays operating in realistic combustion systems, the initial development of liquid jet is often modeled by a set of round liquid blobs injected from the nozzle with similar mass flow rate $[14,73,74]$. These large drops then go through a series of breakup processes mimicing the primary and secondary breakup. This is based on the reasonable assumption that the atomization and fragmentation of liquid jet, blobs or drops are indistinguishable processes within the dense liquid core region near the injector nozzle exit. In this work, spray atomization is modeled by a stochastic model in which the characteristic radius of drops or droplets is assumed to be a time-dependent stochastic variable with a given initial distribution [75],[76]. The breakup of parent drops into daughter droplets is viewed as the temporal and spatial evolution of the distribution function according to the following Fokker-Planck differential equation [76].

$$
\frac{\partial \Im\left(\chi, t^{*}\right)}{\partial t}=-\frac{\langle\varsigma\rangle}{\tau_{b u}} \frac{\partial \Im\left(\chi, t^{*}\right)}{\partial \chi}+\frac{1}{2} \frac{\left\langle\varsigma^{2}\right\rangle}{\tau_{b u}} \frac{\partial^{2} \Im\left(\chi, t^{*}\right)}{\partial \chi^{2}} .
$$

The moments $\langle\varsigma\rangle=\int_{-\infty}^{0} \varsigma S(\varsigma) d \varsigma$ and $\left\langle\varsigma^{2}\right\rangle=\int_{-\infty}^{0} \varsigma^{2} S(\varsigma) d \varsigma$ are the first two moments of the breakup intensity spectrum, which is assumed to be Gaussian. The breakup frequency, $\tau_{b u}=\sqrt{3} \sqrt{\frac{\rho_{l}}{\rho_{g}}} \frac{r_{p}}{u_{r e l_{b u}}}$ is based on the Lagrangian relative velocity fluctuations for the droplet, $u_{r e l_{b u}}[51]$.

The following spherically-symmetric heat and mass transfer equations inside individual droplets are solved for density, energy and species mass fractions $[77,78,51]$.

$$
\begin{gathered}
\frac{\partial \rho_{l}}{\partial t}+\frac{1}{r^{2}} \frac{\partial}{\partial r}\left(r^{2} \rho_{l} v_{l}\right)=0 \\
\frac{\partial\left(\rho_{l} \phi_{l \alpha}\right)}{\partial t}+\frac{1}{r^{2}} \frac{\partial}{\partial r}\left(r^{2} \rho_{l} v_{l} \phi_{l \alpha}\right)=\frac{1}{r^{2}} \frac{\partial}{\partial r}\left(r^{2} \rho_{l} D_{l} \frac{\partial \phi_{l \alpha}}{\partial r}\right) \\
\frac{\partial\left(\rho_{l} T_{l}\right)}{\partial t}+\frac{1}{r^{2}} \frac{\partial}{\partial r}\left(r^{2} \rho_{l} v_{l} T_{l}\right)=\frac{1}{c_{p} r^{2}} \frac{\partial}{\partial r}\left(r^{2} \lambda_{l} \frac{\partial T_{l}}{\partial r}\right) .
\end{gathered}
$$


The mass and energy conservation equations provide the necessary droplet interface parameters such as radial velocity $v_{l s}$, and species mass fraction and temperature gradients at the droplet surface. Moreover, global droplet mass and energy changes such as the total change in the droplet internal energy $d(m e)_{p} / d t$ are also obtained by integrating these equations. The internal droplet circulation, caused by the relative motion of gas and liquid, and its effect on the droplet variables are accounted for by changing the effective mass and thermal liquid diffusivities [79]. For a multi-component droplet, the mass flux of species $\alpha$ at the droplet interface is defined based on the species mass fraction on liquid side of the droplet surface, $\phi_{l s \alpha}$, the droplet radius, $r_{s}$, the species diffusivity in the gas, $D_{g \alpha}$, the species Sherwood number $S h_{\alpha}$ and the Splading mass transfer number, $B_{M \alpha}$ as:

$$
\dot{m}_{\alpha}=\phi_{l s \alpha} 2 \pi r_{s} \rho_{g s} D_{g \alpha} S h_{\alpha} \ln \left(1+B_{M \alpha}\right),
$$

The Spalding mass transfer number relates the liquid and gas interface mass fractions $\left(\phi_{l s \alpha}\right.$ and $\left.\phi_{g s \alpha}\right)$ to the free stream (with respect to droplet) mass fraction $\phi_{\infty \alpha}$ as:

$$
B_{M \alpha}=\frac{\phi_{g s \alpha}-\phi_{\infty \alpha}}{\phi_{l s \alpha}-\phi_{g s \alpha}}
$$

The Ranz-Marshall correlations for Nusselt and Sherwood numbers are used and to account for the surface blowing effect, the film correction proposed by Abramzon and Sirignano [79] is incorporated.

The surface regression rate is found by summing the mass flux over all species

$$
\left(\dot{r}_{s}-v_{l s}\right)=\frac{\rho_{g s} \sum_{\alpha} \phi_{l s \alpha} D_{g \alpha} S h_{\alpha} \ln \left(1+B_{M \alpha}\right)}{2 r_{s} \rho_{l s}} .
$$

The mass conservation for species $\alpha$ and energy balance at droplet interface are governed by the following equations.

$2 r_{s} \rho_{l s}\left(v_{l s}-\dot{r}_{s}\right)\left(\phi_{g s \alpha}-\phi_{l s \alpha}\right)+\left.2 r_{s} \rho_{l s} D_{l s} \frac{\partial \phi_{\alpha}}{\partial r}\right|_{l s}-\rho_{g s} D_{g \alpha} S h_{\alpha} \ln \left(1+B_{M \alpha}\right)=0$
$2 r_{s} \sum_{i} L_{v \alpha} \rho_{l s}\left[\left(\dot{r}_{s}-v_{l s}\right) \phi_{l s \alpha}+\left.D_{l \alpha} \frac{\partial \phi_{\alpha}}{\partial r}\right|_{l s}\right]-\left.2 r_{s} \lambda_{l} \frac{\partial T}{\partial r}\right|_{l s}+\lambda_{g} N u\left(T_{g \infty}-T_{s}\right)=0$. 
The effects of droplets on the carrier gas are included via a series of source/sink terms in the gas continuity, momentum, energy and scalar equations and also in the subgrid kinetic energy equations. These source terms are evaluated by volumetric averaging and interpolation of the Lagrangian variables from all droplets in each cell as:

$$
\begin{gathered}
\left\langle\dot{S}_{\text {Mass }}^{s p}\right\rangle_{l}=-\sum_{p}^{N_{p, c e l l}} \frac{w_{p}}{\Delta V} \dot{m}_{p} \\
\left\langle\dot{S}_{m i}^{s p}\right\rangle_{l}=-\sum_{p}^{N_{p, c e l l}} \frac{w_{p}}{\Delta V}\left[m_{p} \frac{d u_{p i}}{d t}+\dot{m}_{p} u_{p i}\right] \\
\left\langle\dot{S}_{E}^{s p}\right\rangle_{l}=-\sum_{p}^{N_{p, c e l l}} \frac{w_{p}}{\Delta V}\left[\frac{d(m e)_{p}}{d t}+\frac{1}{2} \dot{m}_{p} \mathbf{u}_{p}^{2}+m_{p} \mathbf{u}_{p} \cdot \frac{d \mathbf{u}_{p}}{d t}\right] \\
\left\langle\dot{S}_{\alpha}^{s p}\right\rangle_{l}=-\sum_{p}^{N_{p, c e l l}} \frac{w_{p}}{\Delta V} \dot{m}_{\alpha} \\
\left\langle\dot{S}_{k s g s}^{s p}\right\rangle_{l}=\left(\left\langle\dot{S}_{m i}^{s p}\left\langle u_{i}\right\rangle_{L}\right\rangle_{l}-\left\langle\dot{S}_{m i}^{s p}\right\rangle_{l}\left\langle u_{i}\right\rangle_{L}\right)-\left(\left\langle\dot{S}_{\text {Mass }}^{s p} k_{t o t a l}\right\rangle_{l}-\left\langle\dot{S}_{\text {Mass }}^{s p}\right\rangle_{l} \frac{\left\langle u_{i}\right\rangle_{L}\left\langle u_{i}\right\rangle_{L}}{2}\right)
\end{gathered}
$$

The Spray source term to enthalpy equation which is needed in the FMDF equation is obtained from the following equation:

$$
\left\langle\dot{S}_{h}^{s p}\right\rangle_{l}=\left\langle\dot{S}_{E}^{s p}\right\rangle_{l}-\left\langle u_{i}\right\rangle_{L}\left\langle\dot{S}_{m i}^{s p}\right\rangle_{l}+\frac{\left\langle u_{i}\right\rangle_{L}\left\langle u_{i}\right\rangle_{L}}{2}\left\langle\dot{S}_{\text {Mass }}^{s p}\right\rangle_{l} .
$$

\section{Results and Discussions}

For the selected experiments considered in this paper, the fuel is normal heptane which is injected with the temperature of $373 \mathrm{~K}$ from a 100 micron nozzle with the injection pressure of $150 \mathrm{MPa}$. This spray is designated as "Spray H" by Sandia National laboratory researchers [80]. The main parameters of Spray $\mathrm{H}$ are listed in Table 1. The effect of injection pressure and nozzle size on the spray induced gas turbulence has been considered in our previous work which was focused on non-reacting sprays [46]. The focus of present work is on temperature and oxygen concentration effects on combusting sprays. The 


\begin{tabular}{|l|l|}
\hline Fuel & n-heptane \\
\hline$T_{\text {fuel }}$ & $363 \mathrm{~K}$ \\
\hline$d_{\text {noz }}$ & $100 \mu \mathrm{m}$ \\
\hline$p_{\text {inj }}$ & $150 \mathrm{MPa}$ \\
\hline$\Delta t_{\text {inj }}$ & $4 \mathrm{~ms}$ \\
\hline$T_{g}$ & $800-1200 \mathrm{~K}$ \\
\hline$\rho_{g}$ & $14.8 \mathrm{~kg} / \mathrm{m}^{3}$ \\
\hline
\end{tabular}

Table 1: Parameters of spray $\mathrm{H}$

rectangular computational domain used in the simulations covers the entire axial length of the chamber, but in other directions it extends only to half of the chamber size for computational efficiency. Free stream boundary conditions are applied along these directions. The LES grid size is $0.2 \mathrm{~mm}$ which is uniform in axial direction but stretches in other directions outside of the spray and flame zones. About 20 million finite difference grid points and 120 million Monte Carlo particles are used in our simulations. In contrast to most spray models, there are no adjustable coefficients in our spray model. However, for the initial size and velocity of the injected liquid blobs or drops, the experimentally measured area contraction and nozzle discharge coefficients are used [81]. It has been shown that the spray model is not sensitive to the grid size and the coefficients in the $k_{s g s}$ equation at high ambient gas temperatures [51]. However, the grid size is the smallest that can be used for the specified nozzle size to capture the maximum achievable turbulent scales in the spray generated flow. The computational domain is massively partitioned in all three dimensions for efficient parallel calculations using 624 parallel cores. The code is highly optimized for efficient parallel processing and communications among three parts of the solver. The first stage of P-URAN for chemistry calculations and load balancing is generally conducted at the initial stage of simulation, when the droplet breakup and evaporation are dominant. This is generally prior to flame 


\begin{tabular}{|l|l|l|l|l|}
\hline Case & $\mathbf{O}_{2}$ & $\mathbf{N}_{2}$ & $\mathbf{C O}_{2}$ & $\mathbf{H}_{2} \mathbf{O}$ \\
\hline 1 & 21 & 69.33 & 6.11 & 3.56 \\
\hline 2 & 15 & 75.15 & 6.22 & 3.63 \\
\hline 3 & 12 & 78.07 & 6.28 & 3.65 \\
\hline 4 & 10 & 80.00 & 6.33 & 3.67 \\
\hline 5 & 8 & 81.95 & 6.36 & 3.69 \\
\hline 6 & 0.0 & 89.71 & 6.52 & 3.77 \\
\hline
\end{tabular}

Table 2: Molar percentages of chamber gas mixture for different initial oxygen concentrations.

ignition. The simulation is initialized using the stagnant chamber conditions for the gas temperature, density and composition. The chamber/ambient gas density and temperature for the base case are $14.8 \mathrm{~kg} / \mathrm{m}^{3}$ and $1000 \mathrm{~K}$. Table 2 lists the initial chamber composition for different oxygen concentrations. The oxygen molar percentage for the base case is $21 \%$ (Table 2).

\subsection{Vapor Mixing in Non-Reacting Sprays}

For the non-reacting n-heptane spray, experimental data for liquid and vapor penetration lengths and fuel mass fraction distributions are available. The liquid and vapor penetrations are defined as the axial distance from the nozzle exit to the points where the liquid volume fraction and vapor mass fraction drop to $0.1 \%$ of their initial values [80]. The liquid and vapor penetration lengths predicted by LES/FMDF for the case with ambient density and temperature of $14.8 \mathrm{~kg} / \mathrm{m}^{3}$ and $1000 \mathrm{~K}$ and zero oxygen concentration are compared with the experimental data in Figure 1. During the transient period, the fuel liquid and vapor penetration lengths are both over-predicted by the numerical model. This can be attributed to liquid jet model used for the dense and early development part of the spray. After this period, the results agree well with the experimental data. Additionally, the vapor penetration lengths computed from the LES-FD and FMDF-MC parts of the LES/FMDF solver also agree very well with each other. Further comparison of fuel vapors obtained by LES-FD and FMDF-MC 
are made in Figures 2 and 3, where contours and scatter plots of the vapor mass fraction at $\mathrm{t}=2.00 \mathrm{~ms}$ are shown. It is clear that the Eulerian and Lagrangian solvers are very much consistent and therefore numerically accurate. The scatter in Figure 3 at low vapor mass fraction values could be attributed to the slight "over-shoots" in the Eulerian FD data at the tip of fuel vapor jet, where the flow is highly turbulent and fluctuating. The more diffused behavior of filtered variables computed from MC particles can be mostly attributed to the size of ensemble domain used for particle averaging. The high speed spray vaporization generates a highly unstable jet in the gas which is breaking into turbulent structures downstream of the main liquid jet. The species mass fractions used in the solver are obtained from the FMDF part which is non-diffusive and free from overshoot/undershoot errors usually seen in high order FD simulations with limited grid resolutions.

The simulated and measured radial profiles of azimuthally averaged vapor fuel mass fraction at different axial locations are shown in Figure 4. Again, the computed fuel mass fractions from LES-FD and FMDF-MC are consistent and agree fairly well with the measured values. Figure 4 also shows that the LES/FMDF profiles are narrower and have higher central peak values in comparison to experiment for axial locations close to the nozzle. This can be attributed to the localized spray source terms which are computed at FD grid points with (finite-size) volumetric averaging of the droplet quantities. Further downstream, the numerical values are slightly lower than the experimental ones. This can be related to the narrow upstream jet which becomes unstable faster and grows with a rate slightly higher than the experiment.

\subsection{Reacting Sprays}

The available experimental quantities for the reacting sprays are the ignition delay time and the quasi-steady liftoff length. These global quantities vary significantly as the ambient gas conditions are changed. They are used to assess the global accuracy of the LES/FMDF/Spray model. The definitions for computational ignition delay time and the flame liftoff length have been the subject 
of discussions at the ECN workshops [41]. In particular, several different ignition criteria have been employed to identify the spray ignition in experiments as well as in numerics [1, 41]. However, it is generally agreed that the ignition delay times are not very sensitive to the exact definition as opposed to liftoff length [80], which is dependent on how it is calculated. Here, following Bhattacharjee and Haworth [50] and the ECN recommendations [80], the ignition delay time is defined to be the time from the start of injection to the time when the maximum temperature in the domain exceeds the initial temperature by $400 \mathrm{~K}$. The liftoff length is defined to be the axial distance from the injector at which the computed $\mathrm{OH}$ mass fraction reaches to $2 \%$ of its maximum value for that operating condition.

\subsubsection{Flame Ignition}

There can be different types or modes of ignition in liquid-fuel combustion systems, depending on the relative importance of evaporation time scales and convective and molecular diffusion time scales in the surrounding gas [82]. Three modes of ignition are identified: (1) individual droplet ignition, (2) group ignition around droplets, and (3) spray ignition or global ignition. For high speed evaporating sprays, such as those considered here, the spray ignition mode dominates and the ignition occurs mainly away from the liquid spray. This is due to the fact that the evaporating spray generates a high-momentum, cold, vaporsaturated jet in which the ignition cannot occur. Figures 5 and 6 show the evolution of flame and the overall view of the auto-ignition as captured by $\mathrm{OH}$ species concentration and temperature for the case with gas temperature and density of $\mathrm{T}=1000 \mathrm{~K}, \rho=14.8 \mathrm{~kg} / \mathrm{m}^{3}$ and $21 \%$ oxygen concentration (base case). It is clear that as the induced fuel vapor jet is breaking into smaller eddies and becomes highly turbulent downstream of the liquid spray, the mixing of cold fuel vapor and hot oxidizer gas becomes so significant that the gas mixture can ignite. The presence of localized ignition kernels in spray combustion was suggested by Sato et al. [83], who indicated that the ignition occurs in the stagnation region of the fuel vapor tip. On the other hand, Edwards et 
al. [84] observed that the formation and shedding of eddies along the induced vapor-saturated gas jet provide a suitable environment for local mixing and combustion away from the main high-momentum jet. These eddies serve as a medium in which the chemistry and flow time scales can be balanced, leading to the auto-ignition. Consistent with the experimental observations, Figures 5 and 6 show that the ignition kernels (i.e. localized regions of high reactivity and heat release) are created first at the vapor tip, followed by the establishment of the flame front which propagates upstream.

Figure 7 compares the simulated and measured ignition delay times as a function of the gas oxygen concentration for the ambient gas temperature of 1000 $\mathrm{K}$ and as a function of ambient gas temperature for the initial $\mathrm{O}_{2}$ concentration of $21 \%$ in the chamber. Evidently, the numerical results are in good overall agreement with the experimental data. Note that the ignition can be identified as the initiation of rapid (exothermic) reactions or the appearance of a flame in a combustible mixture. However, the ignition delay considered in the spray experiments comprises of a physical delay and a chemical delay. The physical delay deals with the spray breakup, evaporation and vapor mixing times and the chemical delay involves generation of a radical pool and heat release reactions. The physical delay prediction can be assessed via non-reacting spray mixing simulations as conducted in the previous section. The chemical delay depends on the chemical kinetics model as well as the turbulence-chemistry interactions. It has been shown that even with the same reaction mechanism, the chemical delay time is dependent on the turbulence-chemistry interactions which can be well captured by PDF methods in RANS $[13,50]$ and by the FMDF in the present simulations.

As shown in Figure 7a, the hybrid two-phase LES/FMDF model captures very well the variation of ignition delay time with the $\mathrm{O}_{2}$ concentration in the initial gas. From a practical point of view, reductions in the ambient gas oxygen concentration occur in an engine when exhaust gas recirculation (EGR) is used to improve the efficiency and/or to reduce the emission of nitrogen oxides [44]. Expectedly, the ignition delay increases with the decrease in $\mathrm{O}_{2}$ concentration. 
The simulated results in Figure 7b, however, diverge from the experiments at lower initial gas temperatures. At lower temperatures, the flame ignition occurs in two stages. The first stage or the cool flame ignition stage involves the fuel oxidation at a slow rate. This is followed by the second stage of ignition with a "normal" reaction rate and heat release. As the chemistry is relatively slow at lower gas temperatures and the chemical and turbulent time scales are comparable, the solution will be more dependent on the chemical reaction model and its ability to properly capture the cool flame phenomena. The overpredicting trend of the experimental results by LES/FMDF is suggested to be mostly related to the chemistry modeling. The performance of different reaction models at the lower temperature of $\mathrm{T}=800 \mathrm{~K}$ can be assessed by comparing the results obtained with a detailed mechanism for a simple system such as perfectly stirred reactor (PSR) at the same pressure of $3.34 \mathrm{MPa}$ as that of the chamber. The detailed mechanism, developed by the Lawrence Livermore National Laboratory (LLNL), has proven to be a reliable mechanism $[85,86]$. We have compared the results obtained with this mechanism for the PSR with several other reduced and skeletal mechanisms. The considered mechanisms are: (1) the detailed LLNL mechanism [85, 86], (2) a reduced mechanism by LLNL with 160 species and 770 reversible reactions [25], (3) a skeletal mechanism with 88 species and 387 reversible reactions [27], and (4) a skeletal mechanism with 44 species and 112 reversible reactions [26]. Smaller reduced mechanisms, such as the 29 species mechanism of Patel et al. [87] do not perform as well as those above and are not considered. The PSR simulation with detailed chemistry is conducted using commercial CHEMKIN ${ }^{T M}$, while other simulations are performed using ISAT and DDASAC solver. It is clear from Figure 8 that none of reduced and skeletal mechanisms can fully capture the low temperature trend in detailed mechanism, although the predictions of comprehensive reduced mechanism of LLNL are closer to those obtained by the detailed mechanism. The 44 species mechanism gives the highest delay time for the PSR and as the loss of heat and radicals from the ignition kernels in the actual spray has a delaying effect on the ignition, more delay will be observed in spray combustion simula- 
tions when this mechanism is used. The conclusion is that the chemical kinetic model would not significantly improve the spray combustion results unless a comprehensive detailed mechanism is used. We would like to mention here that although the transient low temperature PSR results are different for different mechanisms, the overall agreement among the results obtained with all four mechanisms is reasonably good.

Pickett et al. [88] found out that prior to auto-ignition of the sprayed fuel in the hot gas chamber, a "cool flame" is established near the quasi-steady flame liftoff position, to be stabilized after the auto-ignition. At high ambient gas temperatures $(T>1000 K)$, the "pre-liftoff zone" is generally a low speed recirculation zone or region around the periphery of the intact spray induced gas jet flow. Mixing in this region occurs with a much slower rate compared to that in the turbulent region further downstream such that a highly reactive flame cannot be maintained for the ambient gas temperature of $\mathrm{T}=1000 \mathrm{~K}$. The experimentally observed pre-ignition "cool flame" is qualitatively shown in Figure 9 to be predicted by LES/FMDF. Figure 9 shows the gas temperature and the $\mathrm{OH}$ concentration contours at earlier time of $\mathrm{t}=0.30 \mathrm{~ms}$. It is noted that the $\mathrm{OH}$ concentration in the pre-liftoff region is much smaller than that in the auto-ignition kernels, formed just before the main flame ignition. To show the significance of the chemical ignition delay and the reaction model in the simulated burning spray, LES/FMDF results obtained with a fast global chemistry model are also considered in this paper. With a simple global reaction mechanism [89], there is virtually no chemical ignition delay and the only observed delay is due to physical delays by evaporation and mixing. Because of absence of $\mathrm{OH}$ radical in the global mechanism, the appearance of "high" temperature values in the flow is inferred as the ignition. The temperature of $\mathrm{T}=2200 \mathrm{~K}$ is considered to be "high". Figure 10 shows that with a global mechanism, the low speed recirculation zones on the periphery of the vapor jet are the ignition spots. Simulations with more detailed chemistry, however, delays the auto-ignition and moves it away from the main spray, where the mixing by (spray and evaporation induced) turbulence is very significant. 


\subsubsection{Early Flame Evolution}

The flame ignition can also be studied by scatter plots or "maps" of temperature and equivalence ratio, or mixture fraction. Such illustration is often used to identify the combustion type or regime $[4,50,90]$. The equivalence ratio $\varepsilon$ is computed from the mixture fraction $f$ based on the elemental carbon and hydrogen mass fractions as:

$$
\begin{aligned}
& f=\sum_{\alpha=1}^{N_{s}}\left(M W_{C} n_{C, \alpha}+M W_{H} n_{H, \alpha}\right) \frac{\phi_{\alpha}}{M W_{\alpha}}, \\
& \varepsilon=\frac{f-f_{a}}{1-f}\left(\frac{A}{F}\right)_{s t},
\end{aligned}
$$

where $n_{C, \alpha}$ and $n_{H, \alpha}$ are the number of Carbon and Hydrogen atoms in the species $\alpha,\left(\frac{A}{F}\right)_{s t}$ is the stoichiometric ratio of ambient gas to fuel and $f_{a}$ is the ambient gas mixture fraction. For a typical quasi-steady diesel combustion system [90, 91, 92], one can separate the flow into different regions by using the $\varepsilon-T$ map: low $T$ and low $\varepsilon$ regions correspond to ambient gas condition far from spray and flame; the near injector vapor saturated jet region has low $T$ and high $\varepsilon$; the slow rate mixing regions have low $T$ and variable $\varepsilon$; the rich premixed flame zone in the main flame interior has high $T$ and high $\varepsilon$; and the outer diffusion flame pockets or sheets formed between the products of premixed flame and the ambient gas jet is represented by high $T$ and low $\varepsilon$. Figure 11 shows the $\varepsilon-T$ scatter plots at different times for the base case as obtained from LES/FMDF data. Prior to ignition, before time $\tau_{\text {delay }}=0.57 \mathrm{~ms}$, the temperature of MC particles having equivalence ratios between 1.5 to 1.8 begin to rise till the ignition. The temperature reaches to $2000 \mathrm{~K}$ for the particles with higher equivalence ratios of around 1.8 but rises moderately for equivalence ratios of 1.4 and lower. This shows that the ignition first occurs at the fuel-rich zones with equivalence ratios around 1.8. These zones correspond to turbulent eddies at the tip of vapor jet, where the mixing and chemical time scales reach a balance. However after the ignition, the high temperature gas products quickly spread to other regions in the flow with lower equivalence ratios. By $\mathrm{t}=0.65$ ms, MC particles with $\varepsilon=1$ reach to temperature of $2000 \mathrm{~K}$ and the flame front is gradually formed around the outer diffusion layer between the turbulent 
jet and the ambient gas, where the equivalence ratio is unity or lower. The regions with intermediate temperatures and equivalence ratios correspond to turbulent flow zones, where the fuel from the vapor jet is mixed with the outer high temperature gas. From $\mathrm{t}=0.70 \mathrm{~ms}$ onward, the outer diffusion flame on the fuel vapor and ambient gas sides begins to establish and by $t=1.00 \mathrm{~ms}$ a quasi-steady flame structure is nearly constructed.

In order to further investigate the flame evolution in composition space through the ignition process, the variation of several ignition marker species such as $\mathrm{CH}_{2} \mathrm{O}, \mathrm{HO}_{2}$ and $\mathrm{H}_{2} \mathrm{O}_{2}$ are depicted in Figure 12. Prior to ignition, from t $=0.50$ to $0.60 \mathrm{~ms}, \mathrm{CH}_{2} \mathrm{O}, \mathrm{HO}_{2}$ and $\mathrm{H}_{2} \mathrm{O}_{2}$ concentrations increase and reach their peak values by the ignition time. The peak of precursor species at $\mathrm{t}=0.50 \mathrm{~ms}$ corresponds to the equivalence ratios of 1.5 to 1.8 for which the temperature first begins to rise. On the other hand, it is clear that during the combustion build-up process up to ignition ( $\mathrm{t}=0.50$ to $0.60 \mathrm{~ms}$ ), the precursor species accumulate on the outer diffusion layer when the equivalence ratio is relatively low. This is consistent with the gradual temperature increase in this region till the ignition. After ignition starts, the precursor species begin to decrease, somehow with different rates. By $\mathrm{t}=0.65 \mathrm{~ms}$, the accumulation location of $\mathrm{CH}_{2} \mathrm{O}$ is shifting to regions with higher equivalence ratios from the low equivalence ratio side of the outer diffusion layer. This happens faster for $\mathrm{HO}_{2}$, while $\mathrm{H}_{2} \mathrm{O}_{2}$ is being consumed. From $\mathrm{t}=0.70$ to $1.00 \mathrm{~ms}$, the species concentrations decrease in bulk of the flame, implying that a pseudo-steady flame is forming. However, their peak is gradually shifted to higher equivalence ratio of around 2.5 by $\mathrm{t}=1.00 \mathrm{~ms}$. This shows the formation of reaction zones extending from low equivalence ratio diffusion region on the ambient side to high equivalence ratio diffusion region on the fuel rich side. This is also in accord with the $\varepsilon-T$ map (Figure 11). The role that $\mathrm{CH}_{2} \mathrm{O}$ plays along with $\mathrm{H}_{2} \mathrm{O}_{2}$ and $\mathrm{HO}_{2}$ as a precursor to ignition is consistent with experimental findings [3, 94].

Figure 13 shows the evolution of $\mathrm{OH}$ mass fraction versus equivalence ratio during the ignition period. When the ignition starts, the $\mathrm{OH}$ mass fraction begins to rise in the flame kernels somewhat similar to temperature. Evidently, 
the $\mathrm{OH}$ mass fraction evolve around the stoichiometric (unity) equivalence ratio, even though the auto-ignition occurs on the fuel rich and high equivalence ratio side. This is expected as the $\mathrm{OH}$ radical is the footprint of the reaction zone or flame front rather than the auto-ignition zone [3]. Figures 11 and 13 also show that for the quasi-steady flame, the high temperature regions in the flow nearly overlap with the highest $\mathrm{OH}$ concentration regions.

\subsubsection{Flame Liftoff}

Measurements have shown that the turbulent spray flame is often lifted off from the fuel injector tip. From a practical viewpoint, the interest in flame liftoff in diesel sprays arises because of its correlation with soot concentration $[2,95]$. Figure 14 shows the lifted turbulent flame for the base case at $t=2.0$ $\mathrm{ms}$ as visualized by the instantaneous iso-surfaces of $\mathrm{OH}$ mass fraction. For the base case, the flame liftoff occurs in the region of the flow which is filled by turbulent eddies formed by the breakup of the spray-induced vapor jet, although it occasionally moves upstream to the recirculation zones on the periphery of the vapor jet. As the initial gas temperature and oxygen concentration decrease, the liftoff length increases and the flame moves further downstream into the turbulent zone. On the other hand, the liftoff length decreases and the flame starts to cover the entire low-speed recirculation zone at higher ambient gas temperatures and eventually reaches to the spray at the liquid penetration point. Figure 15 compares the computed and measured liftoff lengths as a function of initial oxygen concentration for the gas temperature of $\mathrm{T}=1000 \mathrm{~K}$ and as a function of initial gas temperature for $21 \%$ oxygen concentration. The reported liftoff length is averaged for a short period of time between 0.5 to $1 \mathrm{~ms}$ since it is highly oscillatory in time. In contrast to the ignition delay time, which is very sensitive to the chemical kinetics and its level of comprehensiveness, the liftoff length is comparatively less sensitive to chemistry and primarily dependent on the flow and turbulence-chemistry interactions. Therefore it is expected that the liftoff length predictions to be accurate, provided that turbulence-chemistry interactions is well predicted. This is supported by the overall good agreement 
of the LES/FMDF results in Figure 15 with the experiment.

As expected, the flame liftoff length increases with the decrease in oxygen concentration. A simple PSR analysis of the reaction mechanisms indicates that the ignition delay time is noticeably underpredicted with the reaction model used in this paper at lower initial oxygen concentrations. This can partially explain why the ignition delay is underpredicted for the lowest oxygen concentration. However, as mentioned before, the loss of heat and radicals from the ignition kernels in spray simulation has a delaying effect, making the underprediction of spray ignition delay time less pronounced (Figure 7a). As fuel vapor mixes better with the ambient oxidizer in turbulent zones, the combustion occurs at a shorter distance from the injector when the model predicts higher reaction rates and hence, the liftoff length will also be underpredicted by the model. Comparison of the LES/FMDF and experimental flame liftoff lengths for the gas with 15\% ambient oxygen level in Figure 16 indicates a good agreement between them at later times. At the onset of ignition, the predicted liftoff length is higher than the experiment but gradually decreases as the flame spreads upstream. This can be attributed to the chemical kinetics model and its behavior in the relatively low temperature turbulent eddies, where the flame ignition occurs.

As mentioned before, the flame liftoff length decreases rapidly with increase in ambient gas temperature. The relatively small liftoff length at high ambient gas temperatures suggests that the flame may significantly interact with small droplets and their evaporation at the tip of liquid spray, creating a hotter environment for faster evaporation. This in turn makes the non-linear liquid droplet interface equations stiff and the numerical simulation of spray more challenging. Nevertheless, we have been able to simulate the spray combustion under all tested conditions. Figure 17 compares the liftoff length, predicted by the LES/FMDF/spray model with global and complex kinetic mechanisms, for different initial gas temperatures with the experimental data. Although the spray combustion results obtained conducted with the global model are rather poor in terms of ignition delay time and the computed liftoff lengths are generally 
shorter than the experiment, the trend in the liftoff length is captured by this model and its predictions become closer to the experiment at higher ambient gas temperatures. The reaction is faster at higher chamber temperatures and is expected to be better predicted by the global model. This comparison indicates that while the global reaction model is not quantitatively accurate, it is still useful for the overall study of the spray and testing of LES/FMDF/Spray submodels [96].

\subsubsection{Consistency of FMDF in Reacting Spray Simulations}

Figure 18 shows the temperature field obtained from the LES-FD and FMDFMC parts of the hybrid LES/FMDF solver with skeletal reaction mechanism at $\mathrm{t}=2.00 \mathrm{~ms}$. It is clear that the temperature in the "flame liftoff region" is much higher than that in the ambient gas and much lower compared to that in the main flame zone. High-speed chemiluminescence imaging [88] showed that high temperature auto-ignition occasionally occurs in kernels upstream of, and detached from, the high temperature reaction zone, suggesting that the liftoff and stabilization of the flame is not controlled by the flame propagation to the vapor saturated jet. As mentioned before, the liftoff region overlaps with the low-speed recirculation zones around the core of vapor jet at intermediate to high chamber temperatures. In these regions, the mixing rate is relatively low and therefore a high temperature flame cannot be established. Because of low speed recirculation and large-scale eddies, mixing time scale is relatively large, therefore the reaction is less effective in establishing a high temperature flame.

It is also clear from the contour plots in Figure 18 that the LES-FD and FMDF-MC parts of the two-phase LES/FMDF solver are consistent and generate similar results at all locations and times. This consistency indicates the numerical accuracy and the reliability of the LES/FMDF model for the spray combustion. Such a good consistency is only possible in the hybrid LES/FMDF model since the reaction terms (regardless of their complexity) appear in closed forms in the FMDF equation. Here, the reaction terms are calculated from the FMDF-MC particles and used in LES-FD. To further investigate the consis- 
tency of the LES-FD and FMDF-MC methods, scatter plots of the filtered gas temperature and filtered fuel vapor mass fraction as obtained from these two parts of the LES/FMDF are also computed and compared in Figure 19. These scatter plots again show a good overall consistency between the LES-FD and FMDF-MC predictions, confirming once again the overall numerical accuracy of the LES/FMDF/Spray model.

In order to investigate the interaction of the spray with the gas scalar field, as represented by the FMDF, conditional mean values of MC particle variables, conditioned on their spray source terms are shown in Figure 20. For the single component fuel, $\left\langle\dot{S}_{F}^{s p}\right\rangle_{l}=\left\langle\dot{S}_{\text {Mass }}^{s p}\right\rangle_{l}$. Therefore, the spray source term in the MC particle fuel mass fraction equation can be written as $\left(d \phi_{F}^{+} / d t\right)^{s p}=$ $\left(1-\phi_{F}^{+}\right)\left\langle\dot{S}_{\text {Mass }}^{s p}\right\rangle_{l} /\langle\rho\rangle_{l}$. Figure 20a indicates that the vapor fuel mass fractions of $\mathrm{MC}$ particles increases with increase in spray source term up to a certain value and then decreases. This shows that MC particles reach a saturation condition because of evaporation. Figure $20 \mathrm{~b}$ shows the conditional mean of enthalpy for MC particles, normalized by the ambient enthalpy as a function of spray enthalpy source term, also normalized by the ambient enthalpy, $\left(d h^{+} / d t\right)_{n}^{s p}=$ $\left(\left\langle\dot{S}_{h}^{s p}\right\rangle_{l}-h^{+}\left\langle\dot{S}_{\text {Mass }}^{s p}\right\rangle_{l}\right) /\langle\rho\rangle_{l} h_{a}$. It is clear that the contribution of spray to enthalpy is mostly negative. This indicates that the spray mostly extracts energy from the gas field. The spray source term to the energy equation comprise of various parts representing the dissipation of droplet drag force, spray/droplet heating and evaporation. The droplet heating and evaporation parts extract energy from the gas, while the dissipation by drag, which is proportional to droplet relative velocity increases the total energy. In the near injector region, a dense pack of very high speed large drops exist for which heat and mass transfers are negligible. However, the contribution of dissipation term is significant in this region of the flow. As a result, the spray enthalpy source term becomes positive and MC particle enthalpy slightly increases, as compared to the ambient gas enthalpy. Further downstream close to the spray, in the spray generated gas jet, the local enthalpy is reduced by the cooling effect of evaporating droplets. At the very tip of the spray, small droplets interact with turbulent eddies in 
which chemical reactions occur. Interestingly, the highest energy extraction of the spray is from the reacting MC particles whose enthalpies are considerably (up to 2.5 times) higher than the ambient enthalpy.

\subsubsection{Flame Structure}

The flame structure in the simulated reacting (n-heptane) sprays is studied here by examining the contour plots of flow variables and maps of equivalence ratio and temperature. Figure 21 illustrates various regions in the reacting spray through iso-level contours of stoichiometric (unity) equivalence ratio and background temperature contours. As the spray breaks and droplets are formed, dispersed, heated and evaporated, the combined effects of droplet drag and evaporated fuel mass generate a high speed main gas jet which becomes turbulent further downstream. The entrainment and mixing in the turbulent jet is strongly affected by the combustion. There is a clear separation between the high temperature and highly turbulent flame with the liquid spray and droplets in the simulated base case. The ambient gas temperature is high enough for the droplets to evaporate and provide the fuel for the flame without needing the main flame heat. Yet the spray is very fast and generates significant gas flow and turbulence that push the flame away from the spray. Consequently, as Figure 21 shows, there is a relatively cool evaporated fuel that separates the main spray from the main flame. The low temperature, vapor fuel rich gas jet breaks into smaller turbulent structures with much more effective mixing by the spray generated gas flow turbulence before disappearing into the complicated flame plume. The instantaneous gas temperature for the base case at different times in Figure 22 shows that the temperature field, although oscillatory and highly turbulent, does not significantly change in time after the ignition, suggesting that the flame stays nearly steady at these times. Similar trends are observed in the $\mathrm{OH}$ radical contours (not shown).

Figure 23 compares contours of $\mathrm{OH}$ radical mass fraction for the two ambient gas temperatures of 850 and $1200 \mathrm{~K}$ with $21 \%$ oxygen concentration. It is clear that at the higher ambient temperature of $\mathrm{T}=1200 \mathrm{~K}$, the flame front is 
shifted upstream to the periphery of the spray induced vapor jet and the most upstream side of turbulent eddies. Due to high energy content of the ambient gas, the turbulent flame requires less gas entrainment. Therefore, the flame plume is smaller and expands less compared to the case with lower ambient gas temperature. The compositional flame structures for the two flames with initial ambient gas temperatures of $\mathrm{T}=850 \mathrm{~K}$ and $\mathrm{T}=1200 \mathrm{~K}$ are compared in Figure 24 by considering the scatter plots of the equivalence ratio versus temperature. Compared to the base case flame with ambient gas temperature of $1000 \mathrm{~K}$ (or the flame with higher ambient gas temperature of $1200 \mathrm{~K}$ ), the lower temperature $(\mathrm{T}=850 \mathrm{~K})$ flame establishes at equivalence ratios lower or around unity on the ambient side of the $\varepsilon-T$ map and hardly reaches equivalence ratios higher than 1.3 on the fuel rich side of the flame. As the initial energy content of the gas is comparatively low at $\mathrm{T}=850 \mathrm{~K}$, the fuel rich areas are not very reactive. On the other hand, at higher ambient gas temperature of 1200 $\mathrm{K}$, the energy content of the gas is high enough for a rich premixed flame to be developed at very high equivalence ratios. This is in addition to the outer diffusion flames and the intermediate premixed flames. At $\mathrm{T}=1200 \mathrm{~K}$, the chemical time scales are relatively small and the mixing and reaction compete with each other. Therefore, there is a considerable interaction between the spray induced gas flow/turbulence and the combustion.

Figure 25 compares the $O_{2}$ mass fraction contours in the chamber for the two initial $\mathrm{O}_{2}$ concentrations of $21 \%$ and $8 \%$. It is clear that the oxygen diminishes due to fast reactions as it entrains to the turbulent flame. However for the lower initial ambient $\mathrm{O}_{2}$ level, the rate of consumption of $\mathrm{O}_{2}$ is comparatively lower. This suggests that more mixing occurs upstream of the flame for the lower $\mathrm{O}_{2}$ concentration. Figures 26, 27 and 28 show the effect of ambient gas oxygen concentration on the physical and compositional flame structures. The iso-surfaces of stoichiometric (unity) equivalence ratio colored by temperature contours for the two oxygen concentrations of $21 \%$ and $8 \%$ are shown in Figure 26. It is inferred from the figure that at lower oxygen concentration, the flame front, identified by the high temperature iso-surfaces of unity equivalence ratio is 
mainly established on the inner side of the periphery of the main flame and due to lack of oxygen does not penetrate significantly to the core of the turbulent flame. Contours of $\mathrm{OH}$ radical for different initial oxygen concentrations in Figure 27 confirm this observation and further indicate that with lower oxygen concentration in the chamber, not only the peak temperature will be lower but the $\mathrm{OH}$ concentration values will also be much lower.

Figure 28 shows the effect of oxygen concentration on the compositional flame structure by comparing the $\varepsilon-T$ map for flames with $8 \%$ and $21 \%$ of $O_{2}$ molar concentrations. Clearly, the effect of oxygen concentration on the flame is very significant, which is expected. It is observed that with lower oxygen concentration, the thickness of the outer diffusion flame on the $\varepsilon-T$ map increases. This is attributed to the fact that with lower ambient oxygen, more mixing is required for the reactions to occur. At high oxygen levels the flame is not very different than an equilibrium flame, even though the finite-chemistry and nonequilibrium effects are still important in some regions of the flow. However, at lower initial $\mathrm{O}_{2}$ level, the non-equilibrium effects are very significant. There are significant convection/mixing and localized flame extinction in different regions of the flow due to turbulence generated by the spray and lack of oxygen. On average, the flame tends to move toward a premixed condition as substantial mixing occurs before combustion in the lower oxygen level case. The main difference between the flame structures for different oxygen concentrations is that many more thermochemical states are accessed by the flame that has lower oxygen concentration, indicating a higher level of turbulence-chemistry interactions. This is also an indication of higher computational cost for the chemistry calculations with ISAT that needs to cover more points in the $N_{s}+1$ dimensional space of scalars.

\section{Summary and Conclusions}

Hybrid two-phase spray LES/FMDF model is used for numerical simulations of evaporating and combusting n-heptane sprays with a 44 species skeletal 
reaction mechanism. The LES/FMDF mathematical/computational methodology has one Eulerian and two Lagrangian parts. The fluid velocity and pressure field is obtained by solving the filtered form of the compressible NavierStokes equations by high-order finite difference methods via a grid-based Eulerian flow solver. A Lagrangian drop/droplet method with a stochastic breakup model is employed for the spray in which the subgrid turbulence effects on the spray/droplets and the modification of droplet and gas aerodynamics by the wake of nearby droplets are included. Finite rate heat and mass transfer between phases are considered by solving the spherically symmetric inner droplet conservation equations together with the liquid-gas interface equations. The scalar field (species mass fractions and temperature) in the gas and all the complex nonlinear reactions are implemented through the two-phase version of the filtered mass density function (FMDF) methodology. The FMDF is obtained by the solution of its transport equation with an efficient stochastic Lagrangian Monte Carlo method with spray effects included. There are two-way interactions between the phases and all the Eulerian and Lagrangian fields are fully coupled. Combustion chemistry calculations are accelerated by using the parallel and optimized version of in situ adaptive tabulation (ISAT) method.

Simulations of high pressure non-reacting and reacting n-heptane sprays are conducted by the LES/FMDF. For the non-reacting spray, liquid and vapor penetration lengths as well as fuel mass fraction profiles are compared with the experiment. For reacting sprays, ignition delay times and flame liftoff lengths for different ambient gas temperatures and oxygen concentrations are compared. Spray simulations conducted with and without combustion indicate that the two-phase LES/FMDF results are consistent and compare well with the available experimental data. Our reacting LES/FMDF results indicate that at lower range of ambient gas temperatures, the flame auto-ignition occurs in turbulent eddies at the tip of spray-induced, vapor-saturated gas jet and the region just before the flame liftoff point is a "low" temperature flame zone which does not reach the high temperatures of the main flame because of cooling effect of spray and strong convection by spray generated flow. It is shown that the flame igni- 
tion first occurs in the fuel rich regions before the flame rapidly propagates into the stoichiometric and fuel lean regions, thereby forming the lifted flame front. The results for a perfectly stirred reactor indicate that the skeletal reaction mechanism is less accurate compared to the detailed mechanism and overpredicts the ignition delay time for the lower ambient gas temperature range. The liftoff length is less sensitive to the chemical kinetics. It is found that at sufficiently high ambient gas temperatures, the quasi-steady flame liftoff lengths predicted by the global chemical kinetic model are comparable with those obtained by the skeletal mechanism. On the other hand, the flame liftoff is shown to be strongly dependent on the spray parameters, gas temperature and oxygen concentration as well as turbulence-chemistry interactions. The LES/FMDF results indicate that the turbulent flame expands less at higher ambient gas temperatures due to less entrainment. As the fuel rich gas mixture is comparatively cooler for the spray injected into lower ambient gas temperature, the flame tends to move toward regions with lower equivalence ratios. The mixing effect on the flame is reduced with an increase in the ambient gas temperature. Detailed analysis of the flame structure shows that the flame tends to move from a diffusion form toward a more premixed one as the oxygen concentration in the ambient gas decreases and the effect of turbulent mixing on the flame is increased. For the flame with lower ambient oxygen concentration, the nonequilibrium effects are significant and the localized flame extinction is frequent due to strong spray generated turbulence and insufficient oxygen.

\section{Acknowledgment}

This work was supported by the US Department of Energy under agreement DE-FC26-07NT43278. Additional support was provided by the Defense Logistics Agency under agreement DFARS-252232-7010. The authors would like to acknowledge the ISAT package received from Professor Stephen Pope. Computational resources are provided by the Stampede supercomputing system at TACC/UT Austin, funded by NSF award OCI-1134872. 


\section{References}

[1] S. K. Aggarwal, Prog. Energy Combust. Sci. 24 (1998) 565-600.

[2] R. Venugopal, J. Abraham, SAE 01-0134, 2007.

[3] E. Mastorakos, Prog. Energy Combust. Sci. 35 (2009) 57-97.

[4] G. Borghesi, E. Mastorakos, R. S. Cant, Combust. Flame 160 (2013) 12541275 .

[5] H. Pitsch, Ann. Rev. Fluid Mech. 38 (2006) 453-482.

[6] D. C. Haworth, Prog. Energy Combust. Sci. 36 (2010) 168-259.

[7] D. C. Haworth, S. B. Pope, Turbulent Combustion Modeling: Advances, New Trends and Perspectives, Springer, 2011, pp. 119-142.

[8] P. Jenny, D. Roekaerts, N. Beishuizen, Prog. Energy Combust. Sci. 38 (2012) 846-887.

[9] N. Patel, S. Menon, Combust. Flame 153 (2008) 228-257.

[10] W. P. Jones, S. Lira, S. Navarro-Martinez, Combust. Flame 159 (2012) 1539-1561.

[11] A. Banaeizadeh, A. Afshari, H. Schock, F. Jaberi, Int. J. Heat Mass Transfer 60 (2013) 781-796.

[12] V. N. Prasad, A. R. Masri, S. Navarro-Martinez, K. H. Luo, Combust. Flame 160 (2013) 2941-2954.

[13] Y. Pei, E. R. Hawkes, S. Kook, Flow, Turbul. Combust. 91 (2013) 249-280.

[14] X. Jiang, G. A. Siamas, K. Jagus, T. G. Karayiannis, Prog. Energy Combust. Sci. 36 (2010) 131-167.

[15] F. A. Jaberi, P. J. Colucci, S. James, P. Givi, S. B. Pope, J. Fluid Mech. 401 (1999) 85-121. 
[16] A. Afshari, F. A. Jaberi, T. I. P. Shih, AIAA J. 46 (2008) 1576-1592.

[17] S. James, F. A. Jaberi, Combust. Flame 123 (2000) 465-487.

[18] M. R. H. Sheikhi, T. G. Drozda, P. Givi, F. A. Jaberi, S. B. Pope, Proc. Combust. Inst. 30 (2005) 549-556.

[19] P. Givi, AIAA J. 44 (2006) 16-23.

[20] V. Raman, H. Pitsch, Proc. Combust. Inst. 31 (2006) 1711-1719.

[21] M. Yaldizli, K. Mehravaran, F. Jaberi, Int. J. Heat Mass Transfer 53 (2010) $2551-2562$.

[22] Z. Li, A. Banaeizadeh, F. Jaberi, Two-phase filtered mass density function for LES of turbulent reacting flows, 2014. J. Fluid Mech. (in review).

[23] T. Lu, C. K. Law, Prog. Energy Combust. Sci. 35 (2009) 192-215.

[24] F. Battin-Leclerc, Prog. Energy Combust. Sci. 34 (2008) 440-498.

[25] R. Seiser, H. Pitsch, K. Seshadri, W. J. Pitz, H. J. Gurran, Proc. Combust. Inst. 28 (2000) 2029-2037.

[26] S. Liu, J. C. Hewson, J. H. Chen, H. Pitsch, Combust. Flame 137 (2004) 320-339.

[27] C. S. Yoo, T. Lu, J. H. Chen, C. K. Law, Combust. Flame 158 (2011) $1727-1741$.

[28] S. B. Pope, Z. Ren, Flow, Turbul. Combust. 82 (2009) 437-453.

[29] J. Y. Chen, W. Kollmann, R. W. Dibble, Combust. Sci. Tech. 64 (1989) 315-346.

[30] T. Turanyi, J. Comput. Chem. 18 (1994) 45-54.

[31] F. C. Christo, A. R. Masri, E. M. Nebot, Combust. Flame 106 (1996) 406-427. 
[32] J. A. Blasco, N. Fueyo, C. Dopazo, J. Ballester, Combust. Flame 113 (1998) 38-52.

[33] S. B. Pope, Combust. Theory Modelling 1 (1997) 41-63.

[34] L. Lu, S. B. Pope, J. Comput. Phys. 228 (2009) 361-386.

[35] J. B. Bell, N. J. Brown, M. S. Day, M. Frenklach, J. F. Grcar, R. M. Propp, S. R. Tonse, Proc. Combust. Inst. 28 (2000) 107-113.

[36] H. Rabitz, O. F. Alis, J. Math. Chem. 25 (1999) 197-233.

[37] R. R. Cao, S. B. Pope, Combust. Flame 143 (2005) 450-470.

[38] M. Embouazza, D. C. Haworth, N. Darabiha, SAE 2002-01-2773, 2002.

[39] L. Lu, S. R. Lantz, Z. Ren, S. B. Pope, J. Comput. Phys. 228 (2009) 54905525.

[40] V. Hiremath, S. R. Lantz, H. Wang, S. B. Pope, Combust. Flame 159 (2012) 3096-3109.

[41] M. Bardi, R. Payri, L. M. Malbec, G. Bruneaux, L. M. Pickett, J. Manin, T. Bazyn, C. Genzale, Atom. Sprays 22 (2012) 807-842.

[42] D. L. Siebers, SAE 980809, 1998.

[43] D. L. Siebers, B. S. Higgins, SAE 2001-01-0530, 2001.

[44] D. L. Siebers, B. S. Higgins, L. M. Pickett, SAE 2002-01-0890, 2002.

[45] L. M. Pickett, C. L. Genzale, G. Bruneaux, L. M. Malbec, L. Hermant, C. Christiansen, J. Schramm, SAE 01-2106, 2010.

[46] A. Irannejad, F. Jaberi, Numerical study of high speed evaporating sprays, 2014. Int. J. Multiphase Flow (in review).

[47] S. Som, S. K. Aggarwal, Combust. Flame 157 (2010) 1179-1193. 
[48] G. Borghesi, E. Mastorakos, C. B. Devaud, R. W. Bilger, Combust. Theory Modelling 15 (2011) 725-752.

[49] C. Bajaj, M. Ameen, J. Abraham, Combust. Sci. Tech. 185 (2013) 454-472.

[50] S. Bhattacharjee, D. C. Haworth, Combust. Flame 160 (2013) 2083-2102.

[51] A. Irannejad, F. Jaberi, Int. J. Multiphase Flow 61 (2014) 108-128.

[52] A. Banaeizadeh, Z. Li, F. A. Jaberi, AIAA J. 49 (2011) 2130-2143.

[53] P. Moin, K. Squires, W. Cabot, S. Lee, Phys. Fluids A 3 (1991) 2746-2757.

[54] S. Ghosal, T. S. Lund, P. Moin, K. Akselvoll, J. Fluid Mech. 286 (1995) 229-255.

[55] F. A. Jaberi, AIAA 99-0199, 1999.

[56] N. Ansari, F. A. Jaberi, M. R. H. Sheikhi, P. Givi, Engineering Applications of Computational Fluid Dynamics, International Energy and Environment Foundation, 2011, pp. 1-22.

[57] C. K. Madnia, F. A. Jaberi, P. Givi, Handbook of Numerical Heat Transfer, John Wiley and Sons Inc., 2006, pp. 167-189.

[58] P. J. Colucci, F. A. Jaberi, P. Givi, S. B. Pope, Phys. Fluids 10 (1998) 499-515.

[59] C. Dopazo, E. E. O’Brien, Combust. Sci. Tech. 13 (1976) 99-122.

[60] E. E. O’Brien, Turbulent Reacting Flows, Springer, 1980, pp. 185-218.

[61] R. Borghi, Prog. Energy Combust. Sci. 14 (1988) 245-292.

[62] S. B. Pope, Prog. Energy Combust. Sci. 11 (1985) 119-192.

[63] C. W. Gardiner, Handbook of stochastic methods for physics chemistry and the natural sciences, corrected, 2nd ed., 1990. 
[64] P. E. Kloeden, E. Platen, Numerical Solution of Stochastic Differential Equations, Springer, 1995.

[65] M. Caracotsios, W. E. Stewart, Comput. Chem. Eng. 9 (1985) 359-365.

[66] C. Crowe, M. Sommerfeld, Y. Tsuji, Multiphase flows with droplets and particles, CRC Press, 1998.

[67] E. Loth, Prog. Energy Combust. Sci. 26 (2000) 161-223.

[68] W. A. Sirignano, Fluid dynamics and transport of droplets and sprays, Cambridge University Press, 2010.

[69] J. Pozorski, S. V. Apte, Int. J. of Multiphase Flow 35 (2009) 118-128.

[70] A. Irannejad, F. Jaberi, SAE 2013-01-1101, 2013.

[71] J. Bellan, K. Harstad, Int. J. Heat Mass Transfer 30 (1987) 1083-1093.

[72] A. B. Liu, D. Mather, R. D. Reitz, SAE 930072, 1993.

[73] M. Gorokhovski, M. Herrmann, Ann. Rev. Fluid Mech. 40 (2008) 343-366.

[74] P. Moin, S. V. Apte, AIAA J. 44 (2006) 698-708.

[75] A. N. Kolmogorov, Dokl. Akad. Nauk. SSSR 31 (1941) 99-101.

[76] M. A. Gorokhovski, V. L. Saveliev, Phys. Fluids 15 (2003) 184-192.

[77] D. J. Torres, P. J. ORourke, A. A. Amsden, Combust. Theory Model. 7 (2003) 67-86.

[78] S. Srivastava, H. Schock, F. Jaberi, SAE 2013-01-1603, 2013.

[79] B. Abramzon, W. A. Sirignano, Int. J. Heat Mass Transfer 32 (1989) 16051618.

[80] L. M. Pickett, Engine combustion network, Sandia National Laboratory, http://www.sandia.gov/ecn/, 2013. 
[81] A. Kastengren, F. Z. Tilocco, C. F. Powell, J. Manin, L. M. Pickett, R. Payri, T. Bazyn, Atom. Sprays 22 (2012) 1011-1052.

[82] H. H. Chiu, T. M. Liu, Combust. Sci. Tech. 17 (1977) 127-142.

[83] J. I. Sato, K. Konishi, H. Okada, T. Niioka, Symposium (Int.) Combustion 21 (1988) 695-702.

[84] C. Edwards, D. Siebers, D. Hoskin, SAE 920108, 1992.

[85] H. J. Curran, P. Gaffuri, W. J. Pitz, C. K. Westbrook, Combust. Flame 114 (1998) 149-177.

[86] M. Mehl, W. J. Pitz, C. K. Curran, Proc. Combust. Inst. 33 (2011) 193-200.

[87] A. Patel, S. Kong, R. Reitz, SAE 2004-01-0558, 2004.

[88] L. Pickett, D. Siebers, C. Idicheria, SAE 2005-01-3843, 2005.

[89] C. K. Westbrook, F. L. Dryer, Combust. Sci. Tech. 27 (1981) 31-43.

[90] J. E. Dec, Proc. Combust. Inst. 32 (2009) 2727-2742.

[91] J. E. Dec, SAE 970873, 1997.

[92] P. F. Flynn, R. P. Durrett, G. L. Hunter, A. O. zur Loye, O. C. Akinyemi, J. E. Dec, C. K. Westbrook, SAE 1999-01-0509, 1997.

[93] R. L. Gordon, A. R. Masri, E. Mastorakos, Combust. Flame 155 (2008) 181-195.

[94] W. O’Loughlin, A. R. Masri, Flow Turbul. Combust. 89 (2012) 13-35.

[95] L. M. Pickett, D. L. Siebers, Combust. Flame 138 (2004) 114-135.

[96] A. Irannejad, F. Jaberi, AIAA 2013-2599, 2013. 


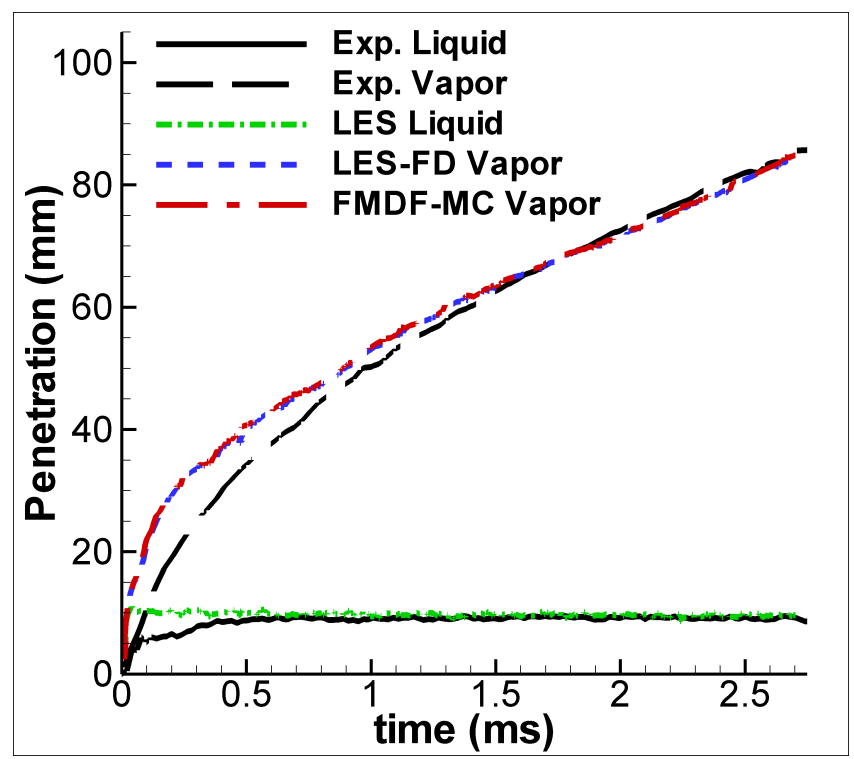

Figure 1: Temporal variations of simulated and measured liquid and vapor penetration lengths for the case with gas temperature and density of $\mathrm{T}=1000 \mathrm{~K}, \rho=14.8 \mathrm{~kg} / \mathrm{m}^{3}$ and oxygen concentration of $0.0 \%$. 


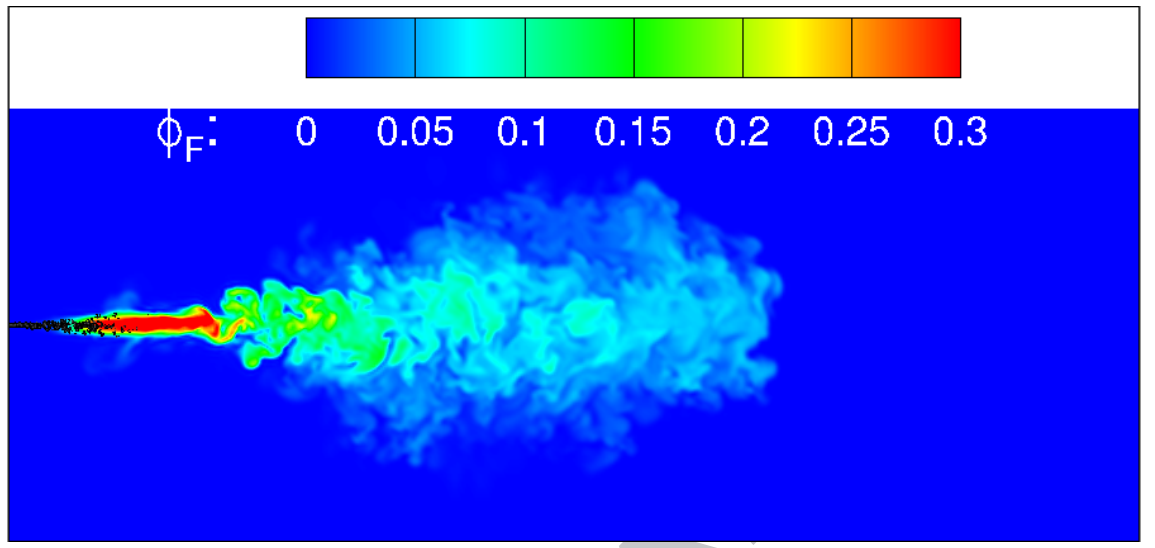

(a)

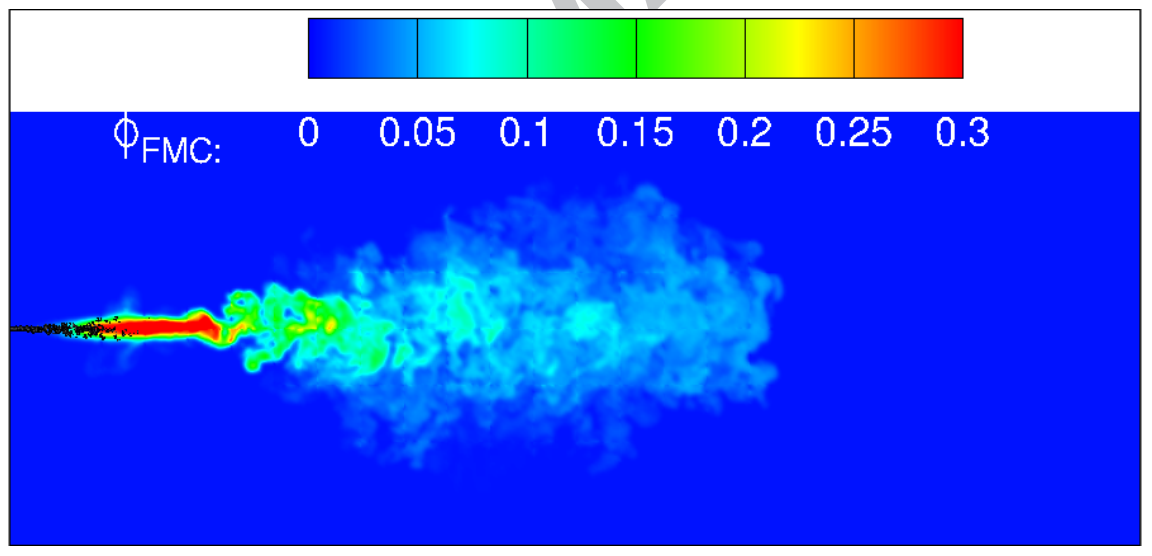

(b)

Figure 2: Fuel vapor mass fraction contours in the non-reacting spray with ambient gas temperature and density of $\mathrm{T}=1000 \mathrm{~K}, \rho=14.8 \mathrm{~kg} / \mathrm{m}^{3}$. (a) LES-FD data, (b) FMDF-MC. Scalar contours in figure 2(a) are obtained from finite difference (FD) LES data, those in 2(b) are obtained from Monte Carlo (MC) FMDF data. 


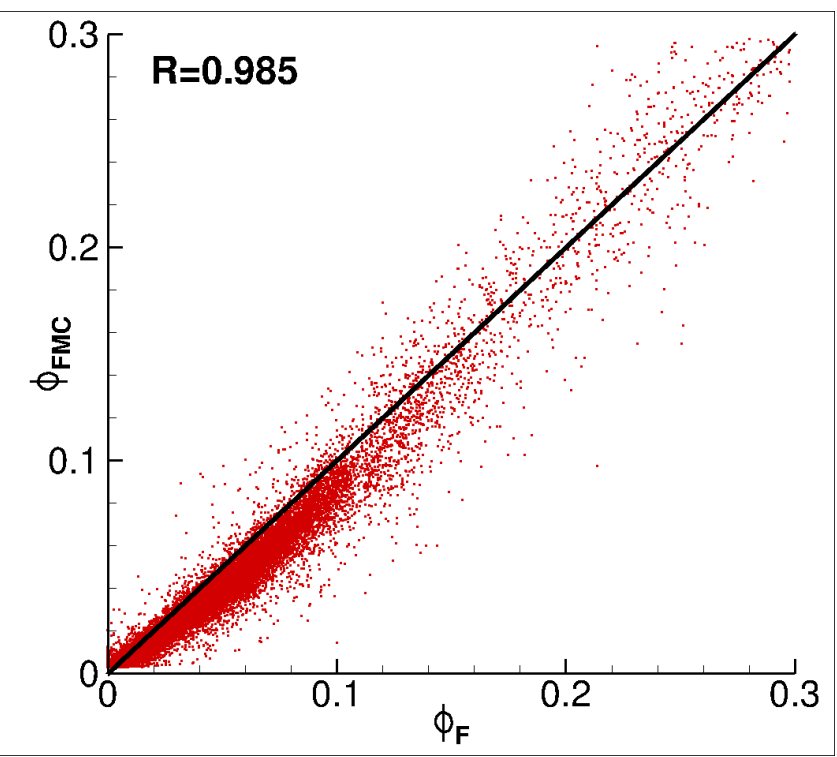

Figure 3: Comparison of vapor mass fraction obtained from LES-FD and FMDF-MC parts of the LES/FMDF solver. 


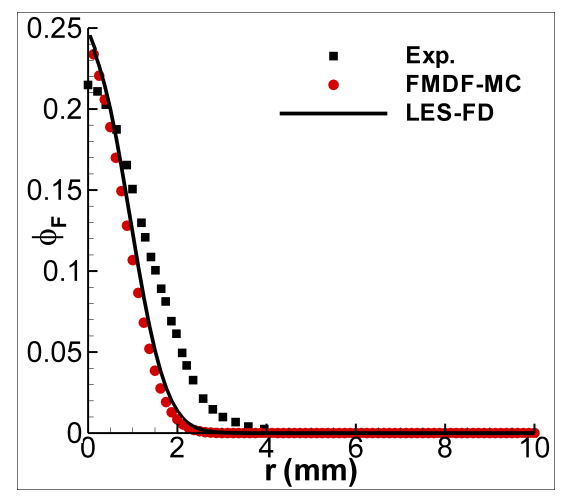

(a)

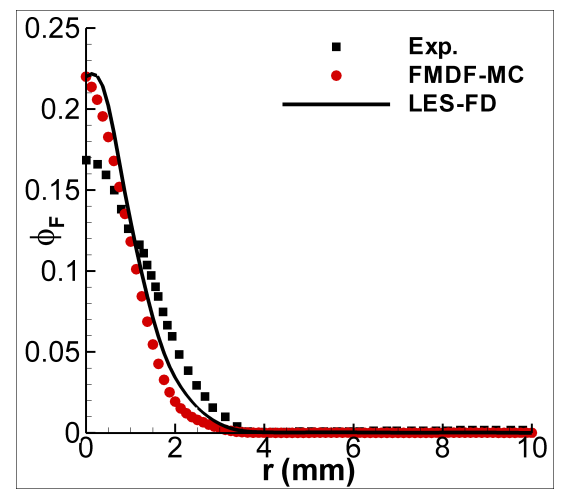

(b)

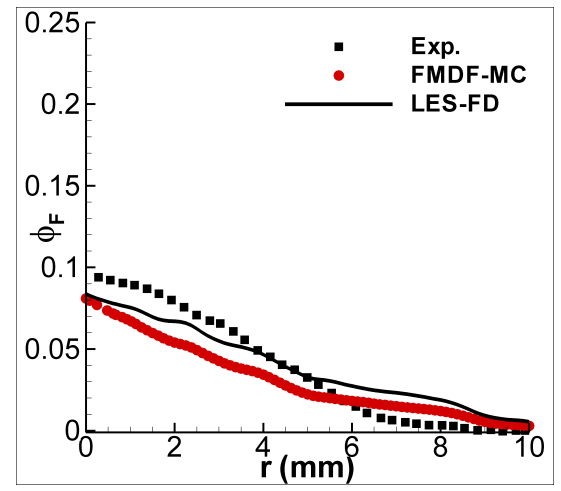

(c)

Figure 4: Radial profiles of simulated and measured fuel mass fraction at different axial locations for the non-reacting evaporating spray. (a) $\mathrm{x}=17 \mathrm{~mm}$, (b) $\mathrm{x}=20 \mathrm{~mm}$, (c) $\mathrm{x}=40 \mathrm{~mm}$. 

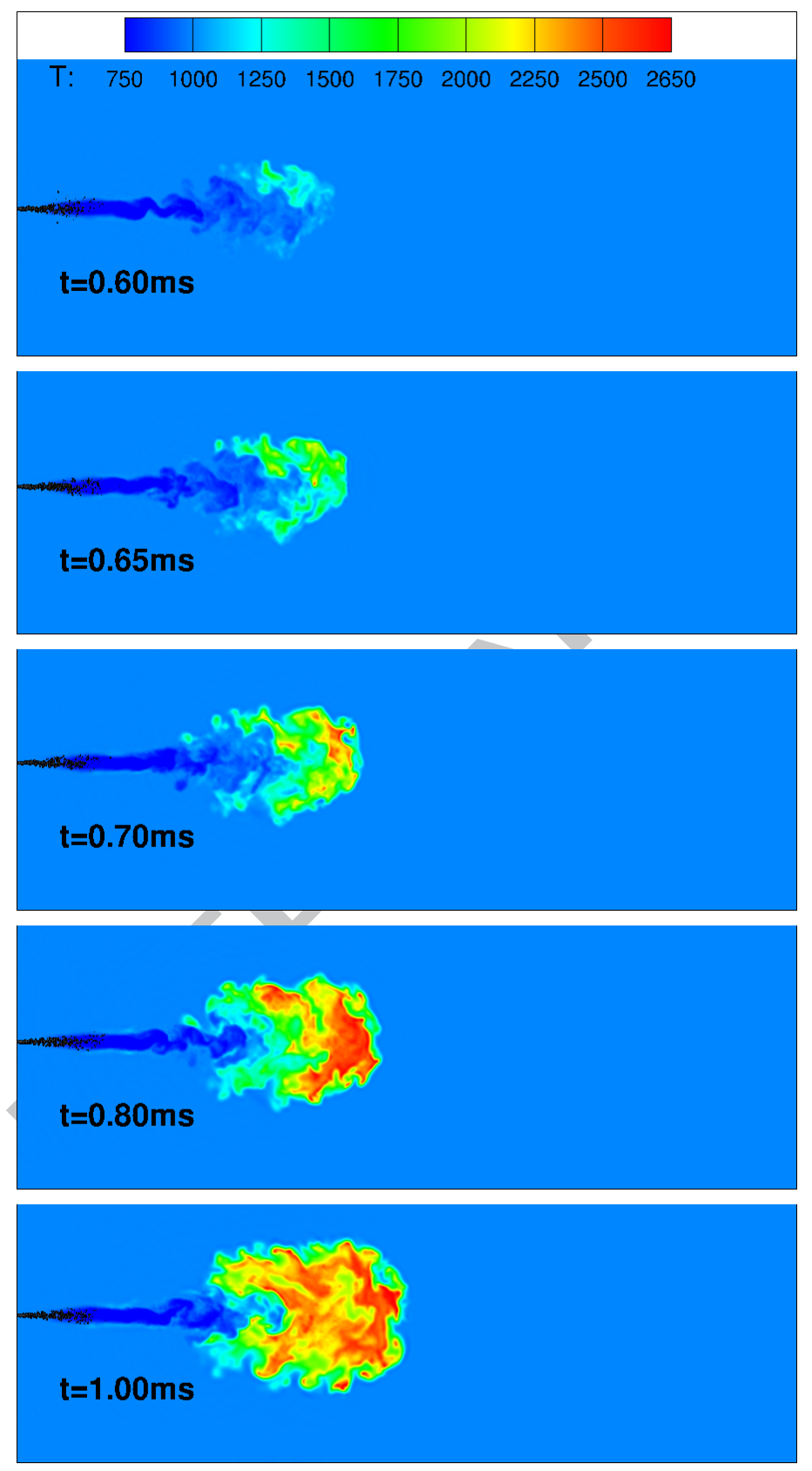

Figure 5: Contours of temperature in the reacting spray case with ambient gas temperature and density of $\mathrm{T}=1000 \mathrm{~K}, \rho=14.8 \mathrm{~kg} / \mathrm{m}^{3}$ and $21 \%$ oxygen concentration (base case) at different times. 

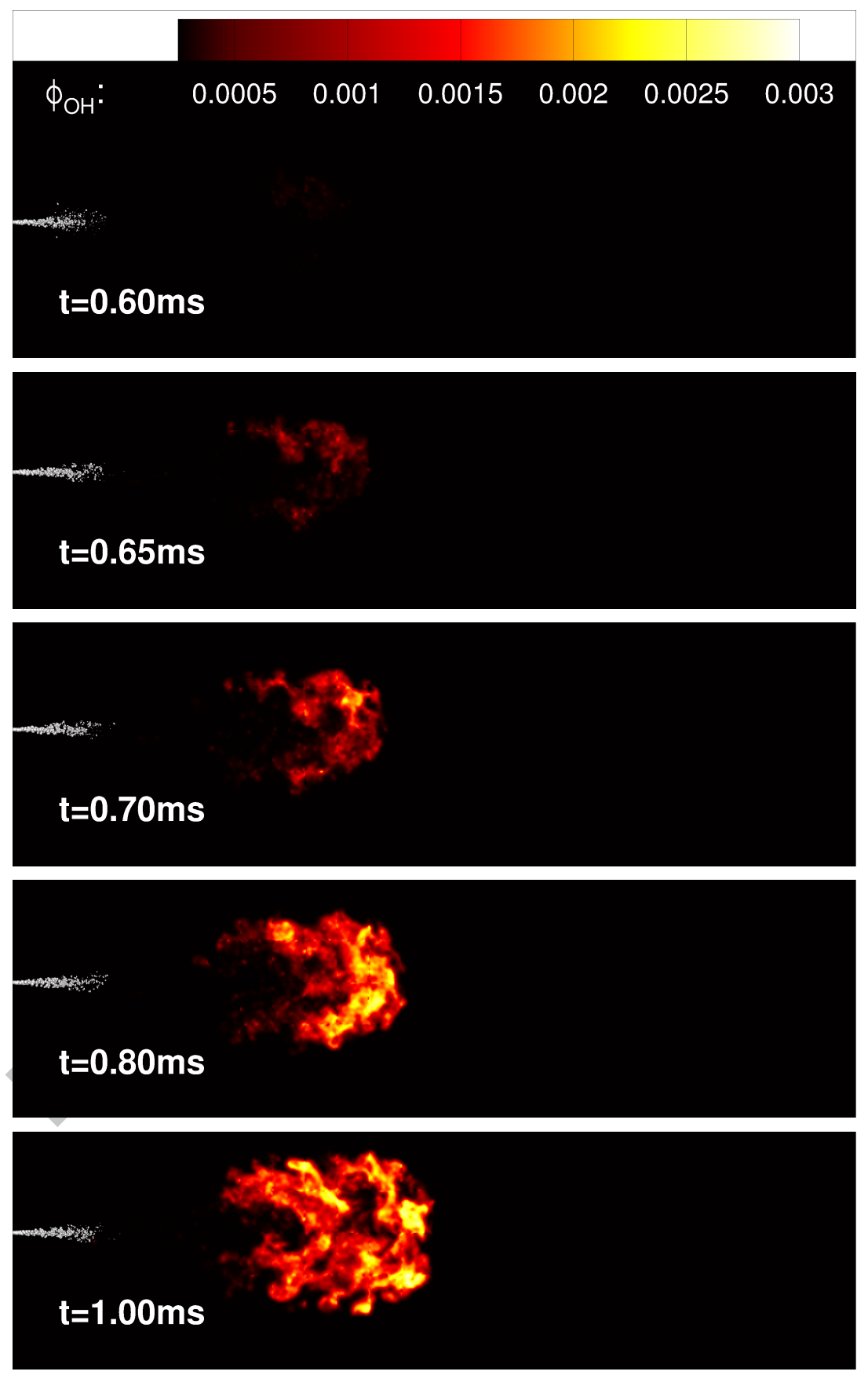

Figure 6: Contours of $\mathrm{OH}$ radical mass fraction in the reacting spray (base case) at different times. 


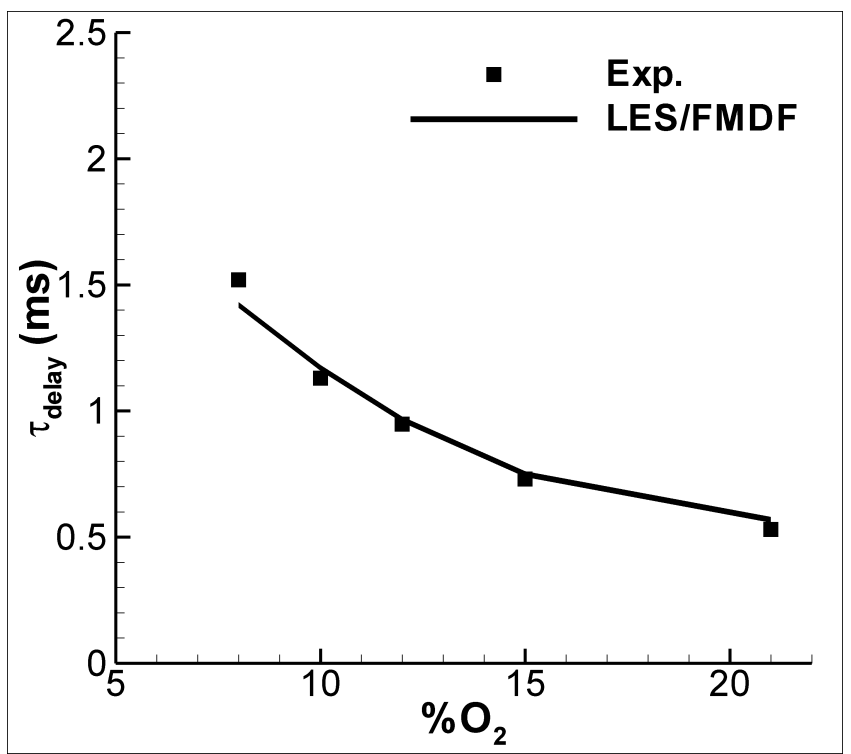

(a)

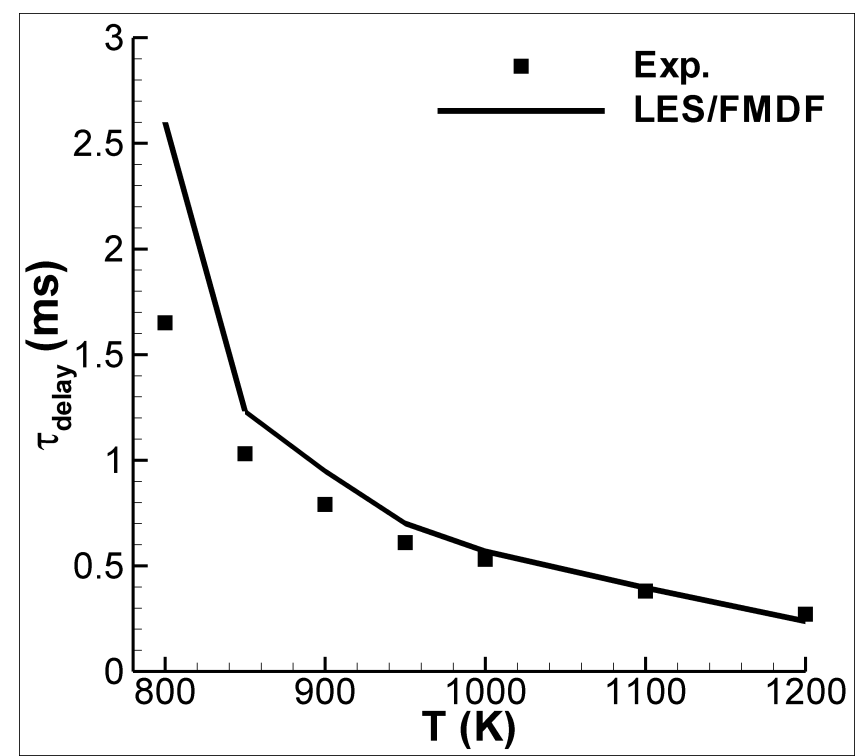

(b)

Figure 7: Simulated and measured ignition delay times (a) versus oxygen molar percentage for $\mathrm{T}=1000 \mathrm{~K}$ and (b) versus chamber temperature for $21 \%$ of $\mathrm{O}_{2}$ concentration. 


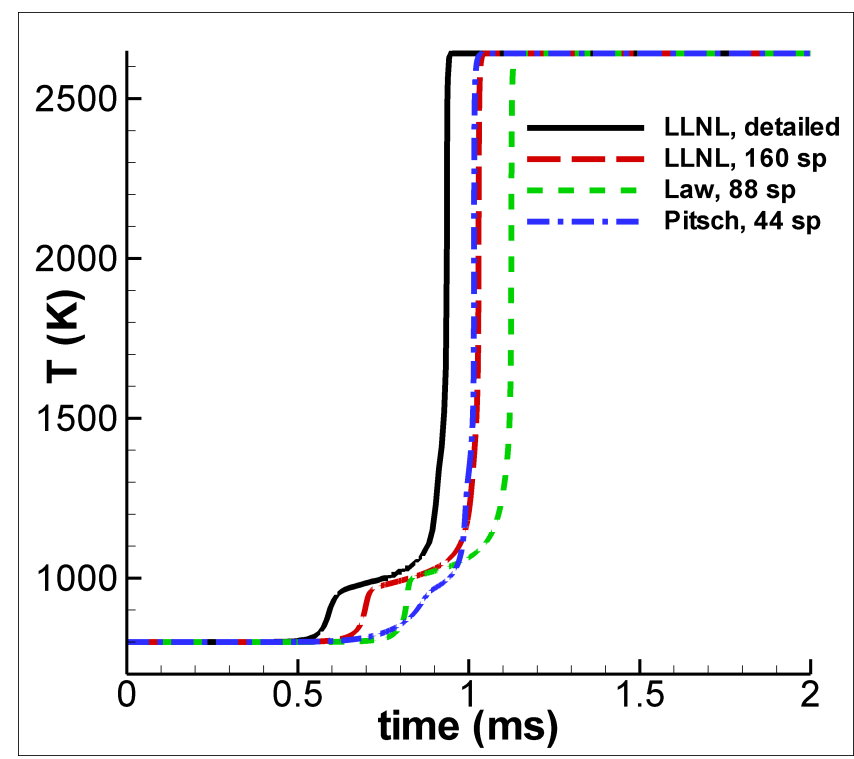

Figure 8: Comparison of temperatures predicted by different reaction mechanisms for low temperature combustion of $n$-heptane in a high pressure perfectly stirred reactor at $\mathrm{T}=800 \mathrm{~K}$ and $\mathrm{P}=3.34 \mathrm{MPa}$. 


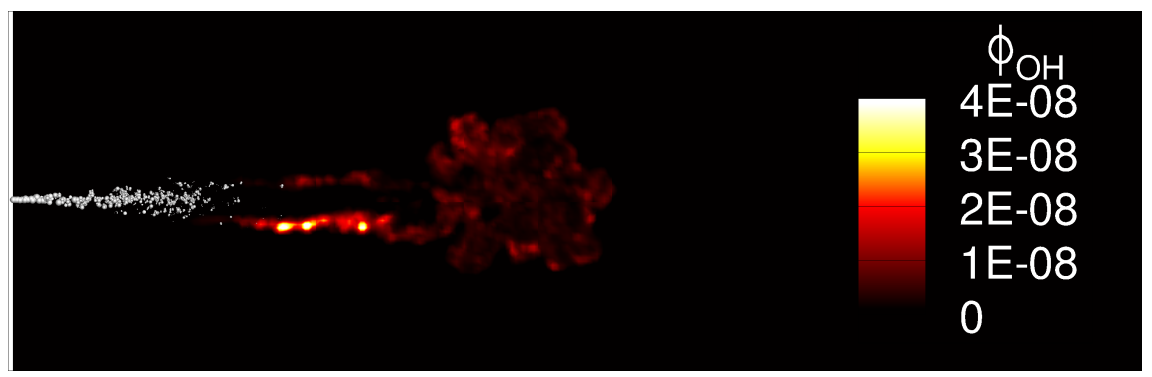

(a)

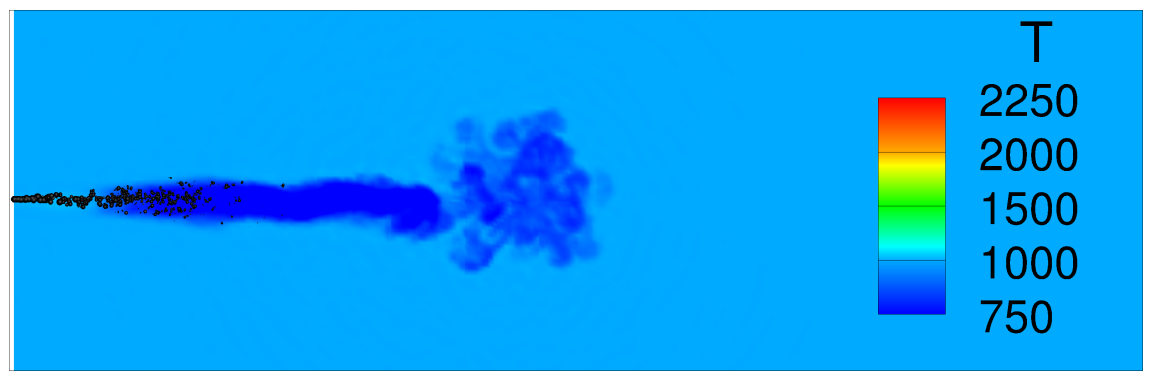

(b)

Figure 9: Visualization of "cool flame" in the near nozzle region prior to auto-ignition at $\mathrm{t}=0.30 \mathrm{~ms}$. (a) $\mathrm{OH}$ mass fraction and (b) temperature contours.
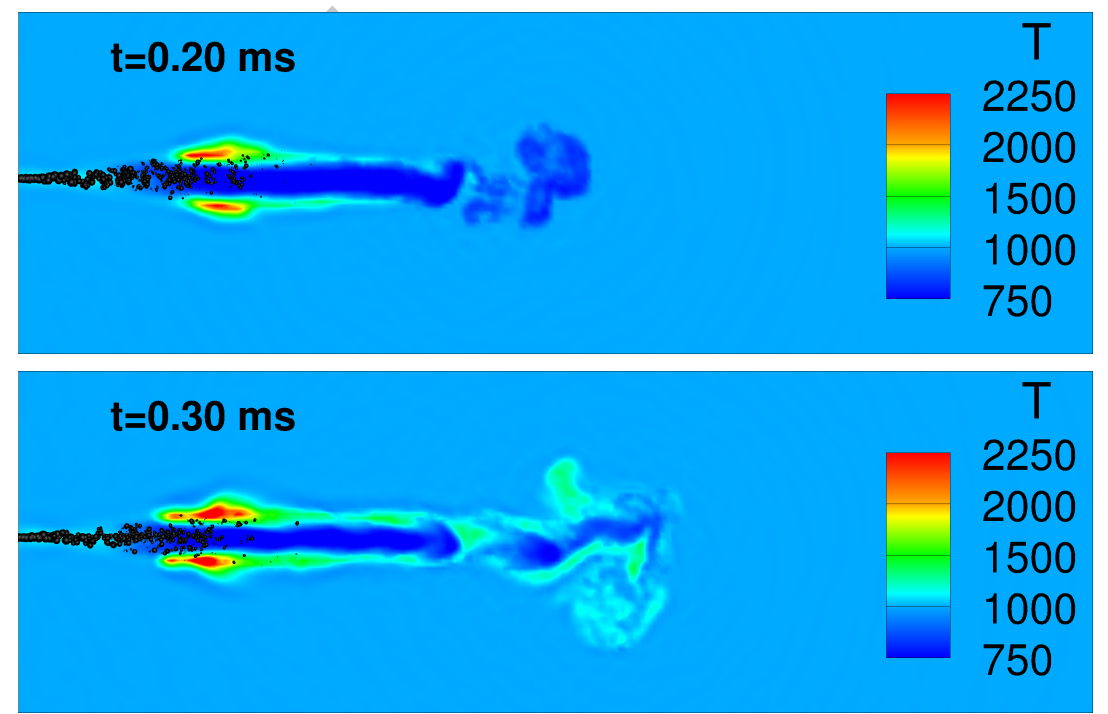

Figure 10: Temperature contours at the onset of ignition at $\mathrm{t}=0.20 \mathrm{~ms}$ and $\mathrm{t}=0.30 \mathrm{~ms}$, obtained by a global one-step reaction model. 


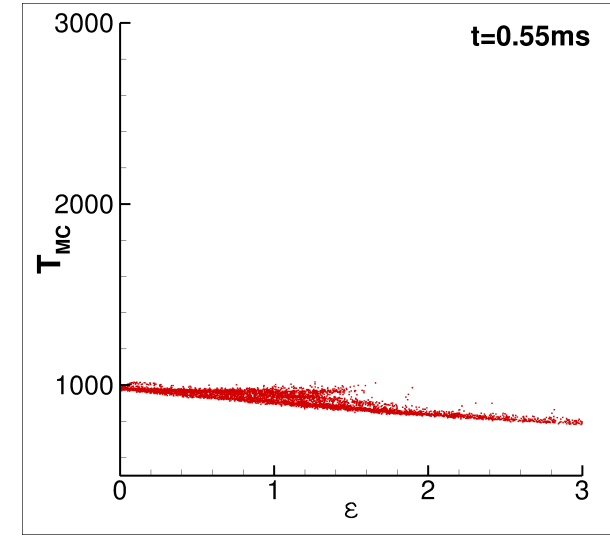

(a)

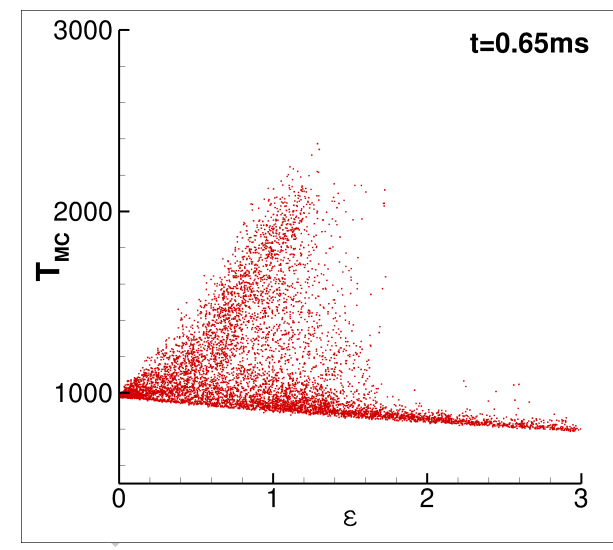

(c)

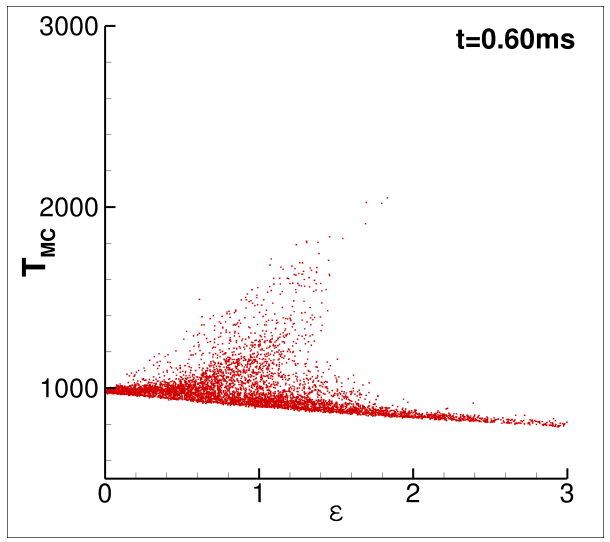

(b)

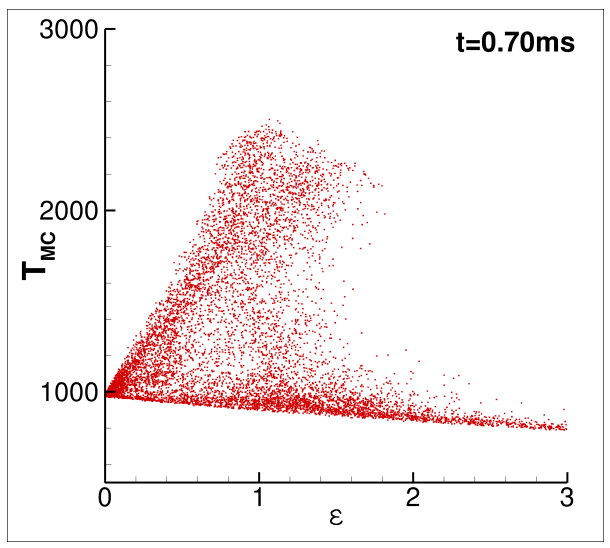

(d)

Figure 11: Map of equivalence ratio versus temperature at different times in the reacting spray, obtained by LES/FMDF/Spray model with skeletal mechanism during auto-ignition. (a) $\mathrm{t}=0.55 \mathrm{~ms}$, (b) $\mathrm{t}=0.60 \mathrm{~ms}$, (c) $\mathrm{t}=0.65 \mathrm{~ms}$, (d) $\mathrm{t}=0.70 \mathrm{~ms}$, (e) $\mathrm{t}=0.80 \mathrm{~ms}$, (f) $\mathrm{t}=1.00 \mathrm{~ms}$. 


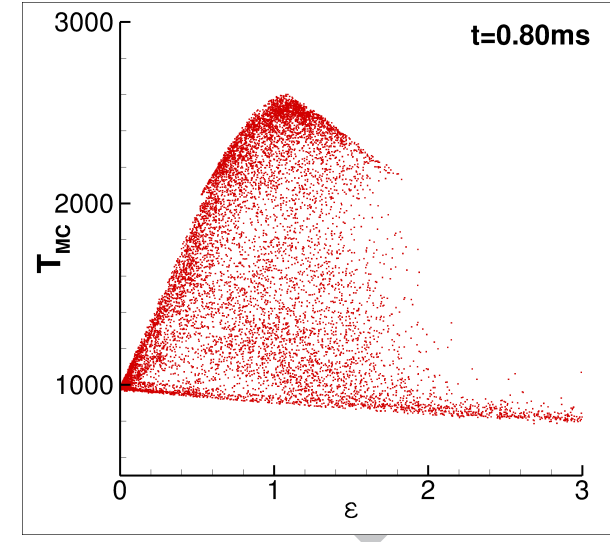

(e)

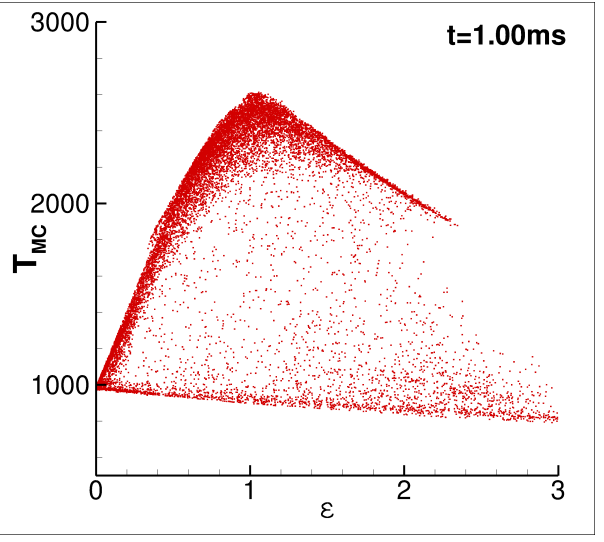

(f)

Figure 11: (cont'd) 

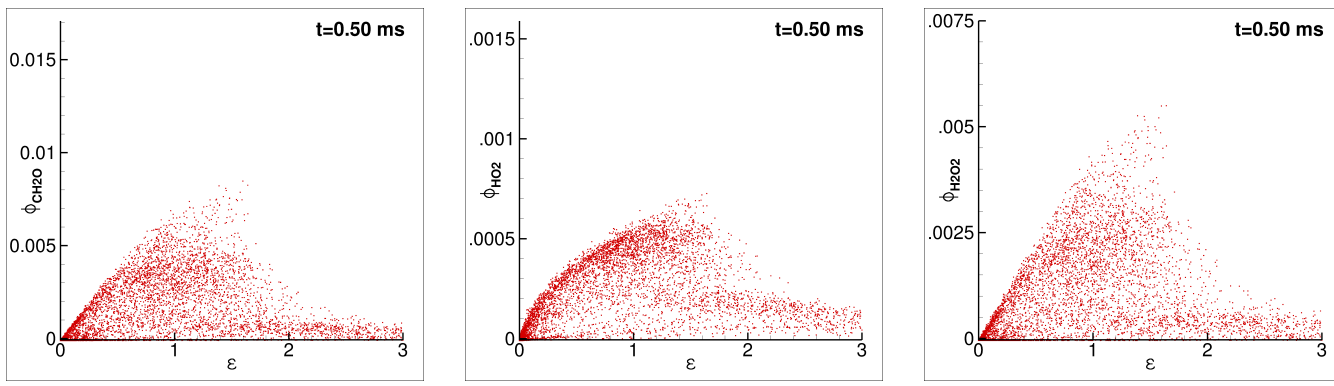

(a)
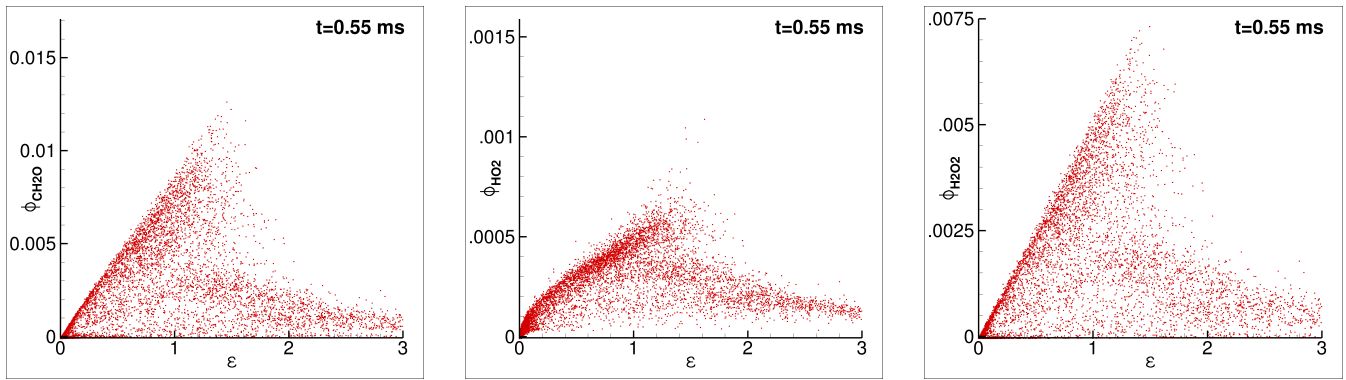

(b)
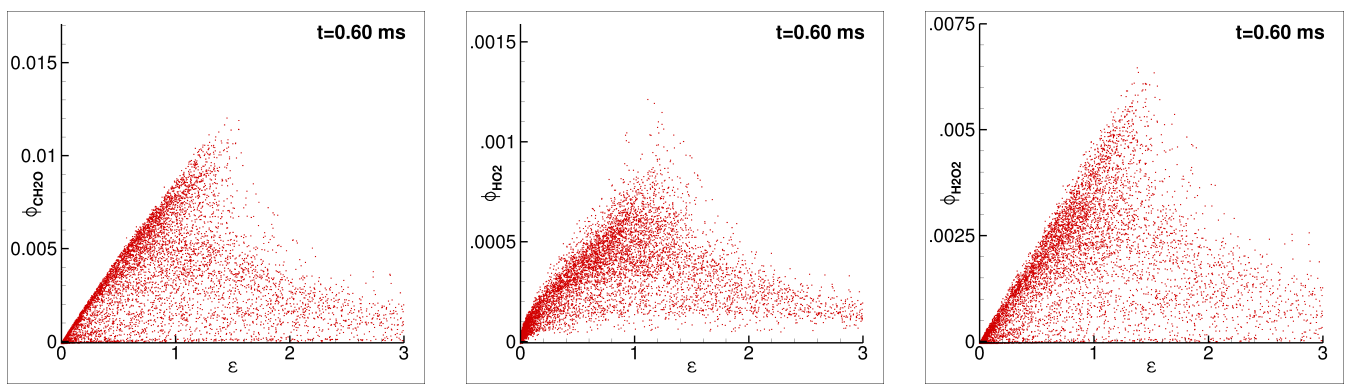

(c)

Figure 12: Map of $\mathrm{CH}_{2} \mathrm{O}, \mathrm{HO}_{2}$ and $\mathrm{H}_{2} \mathrm{O}_{2}$ mass fractions versus equivalence ratio at different times in the reacting spray, obtained by LES/FMDF/Spray model with skeletal mechanism during auto-ignition. (a) $t=0.50 \mathrm{~ms}$, (b) $t=0.55 \mathrm{~ms}$, (c) $t=0.60 \mathrm{~ms}$, (d) $t=0.65 \mathrm{~ms}$, (e) $\mathrm{t}=0.70$ $\mathrm{ms}$, (f) $\mathrm{t}=0.80 \mathrm{~ms},(\mathrm{~g}) \mathrm{t}=1.00 \mathrm{~ms}$. 

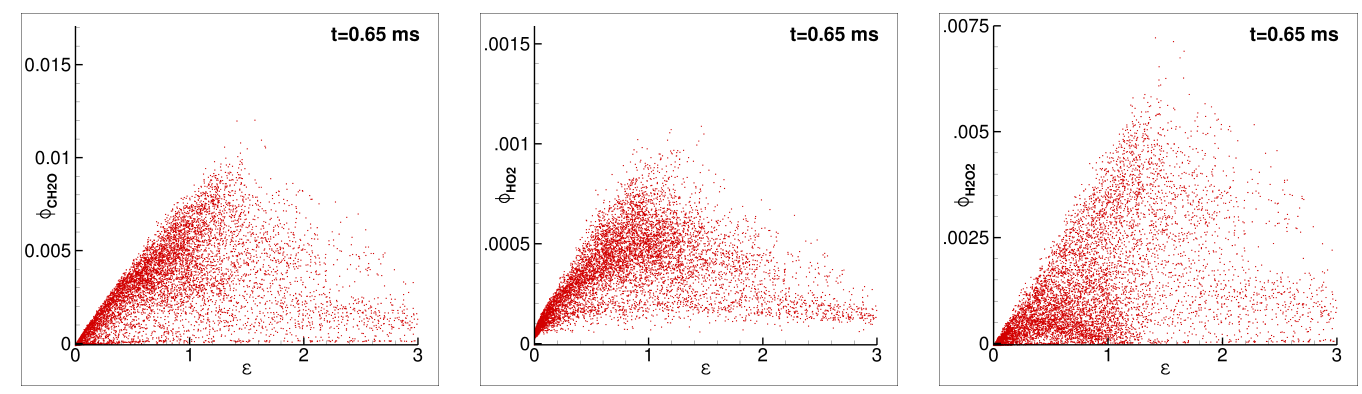

(d)
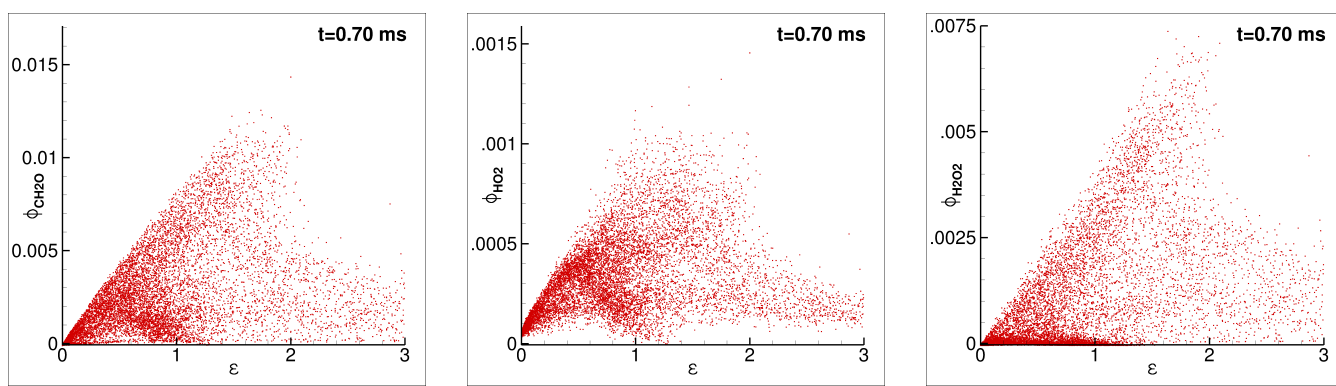

(e)
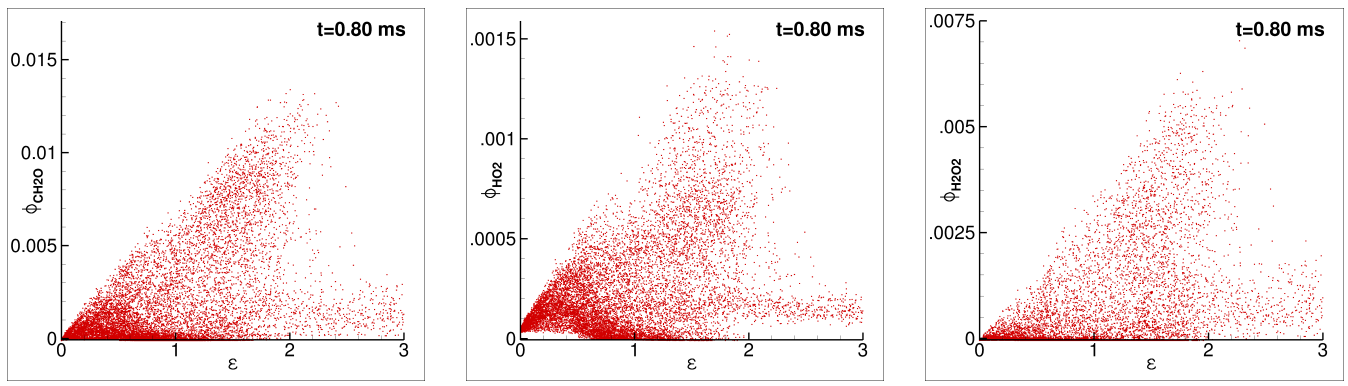

(f)

Figure 12: (cont'd) 

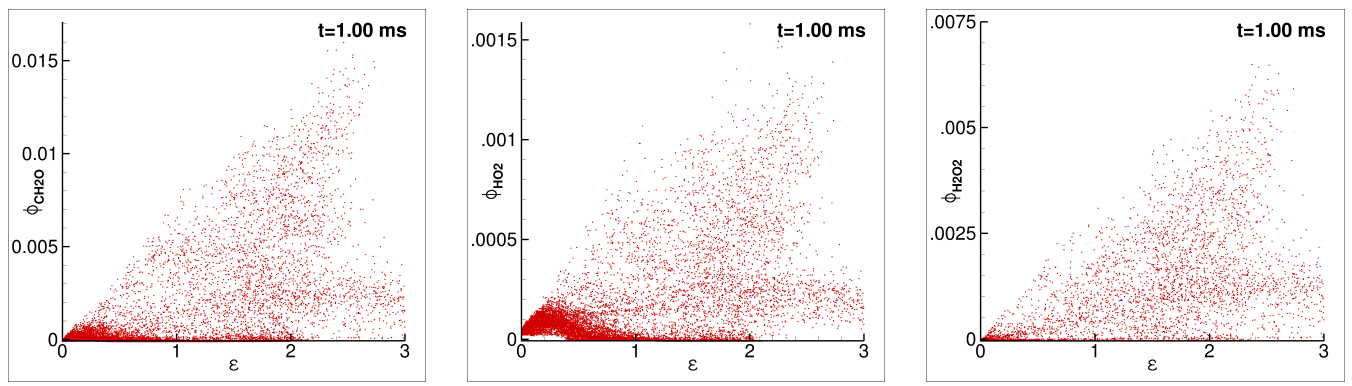

(g)

Figure 12: (cont'd) 


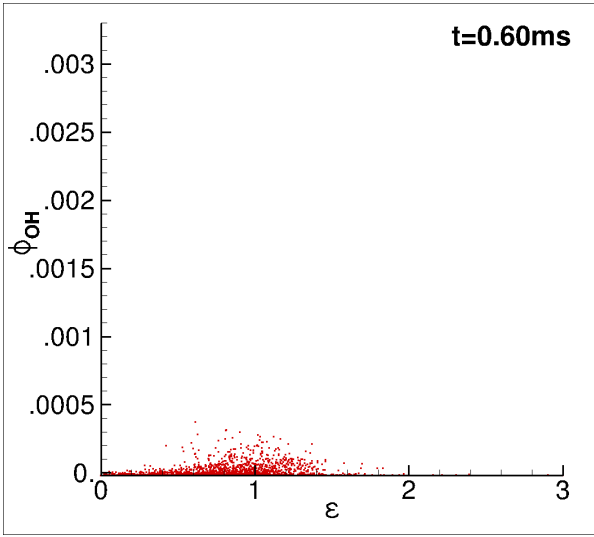

(a)

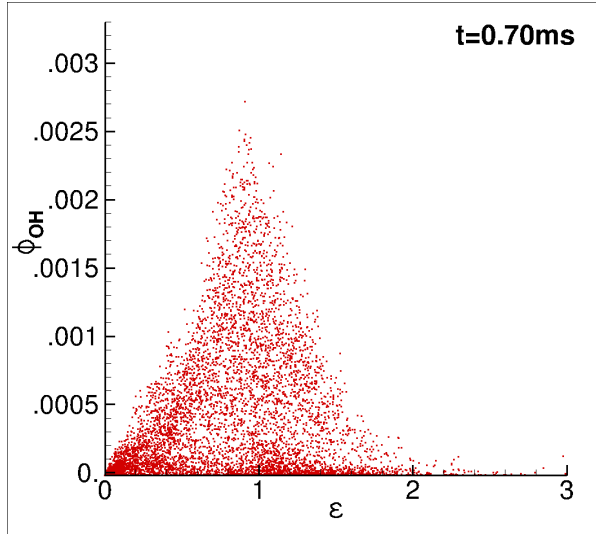

(c)

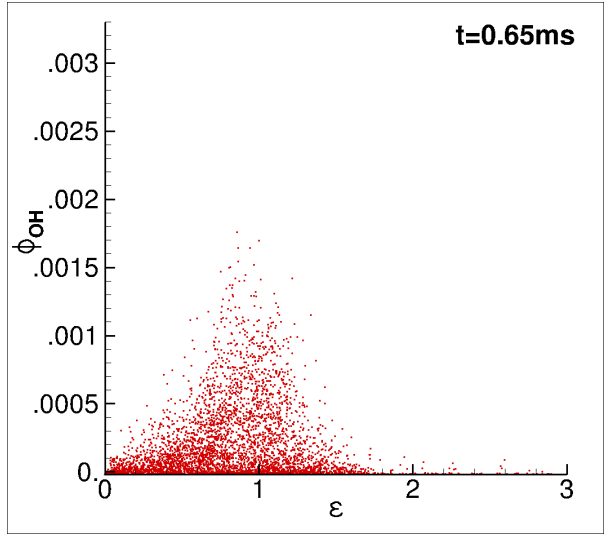

(b)

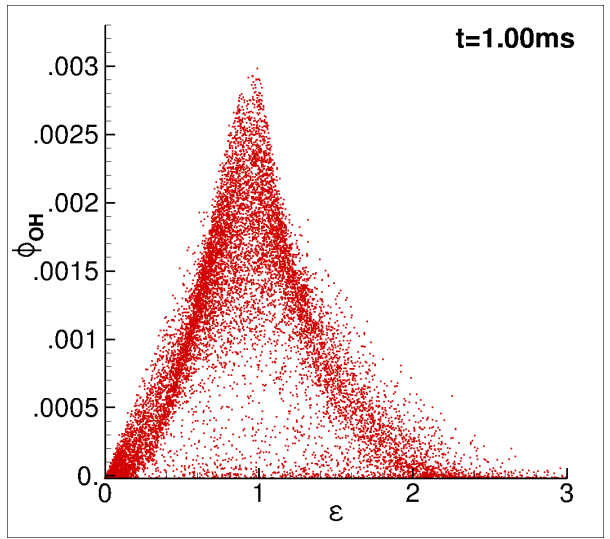

(d)

Figure 13: Map of $\mathrm{OH}$ mass fraction versus equivalence ratio at different times in the reacting spray, obtained by LES/FMDF/Spray model with skeletal mechanism during auto-ignition. (a) $\mathrm{t}=0.60 \mathrm{~ms}$, (b) $\mathrm{t}=0.65 \mathrm{~ms}$, (c) $\mathrm{t}=0.70 \mathrm{~ms}$, (d) $\mathrm{t}=0.100 \mathrm{~ms}$. 


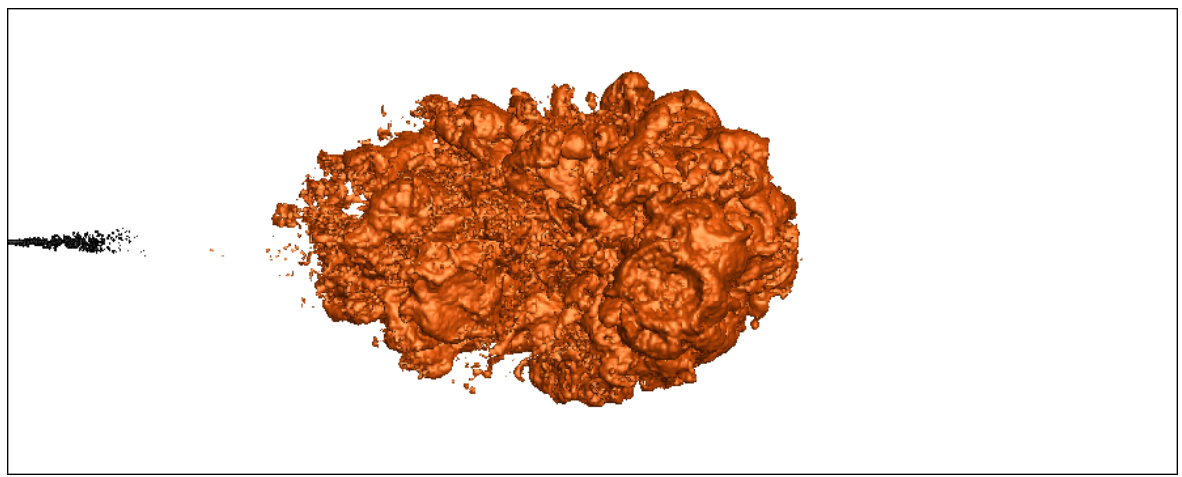

Figure 14: Iso-surfaces of $\mathrm{OH}$ mass fraction in the reacting spray (base case) at $\mathrm{t}=2.00 \mathrm{~ms}$. 


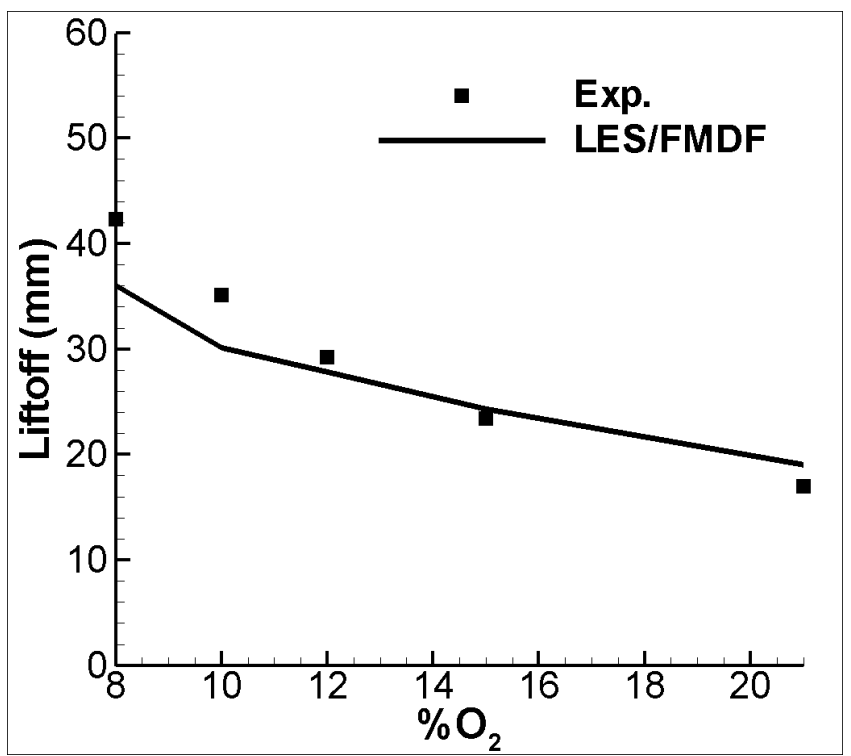

(a)

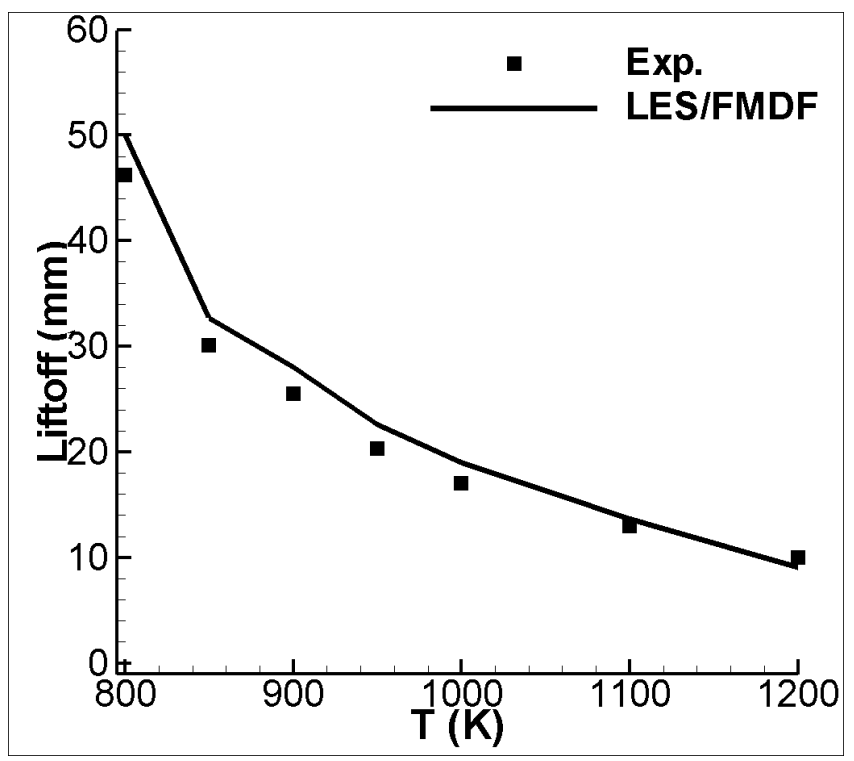

(b)

Figure 15: Comparison of computed flame liftoff length with the experimental data for different chamber gas conditions. (a) liftoff length versus $\mathrm{O} 2$ concentration for $\mathrm{T}=1000 \mathrm{~K}$, and (b) liftoff length versus chamber temperature for $21 \% \mathrm{O}_{2}$ concentration. 


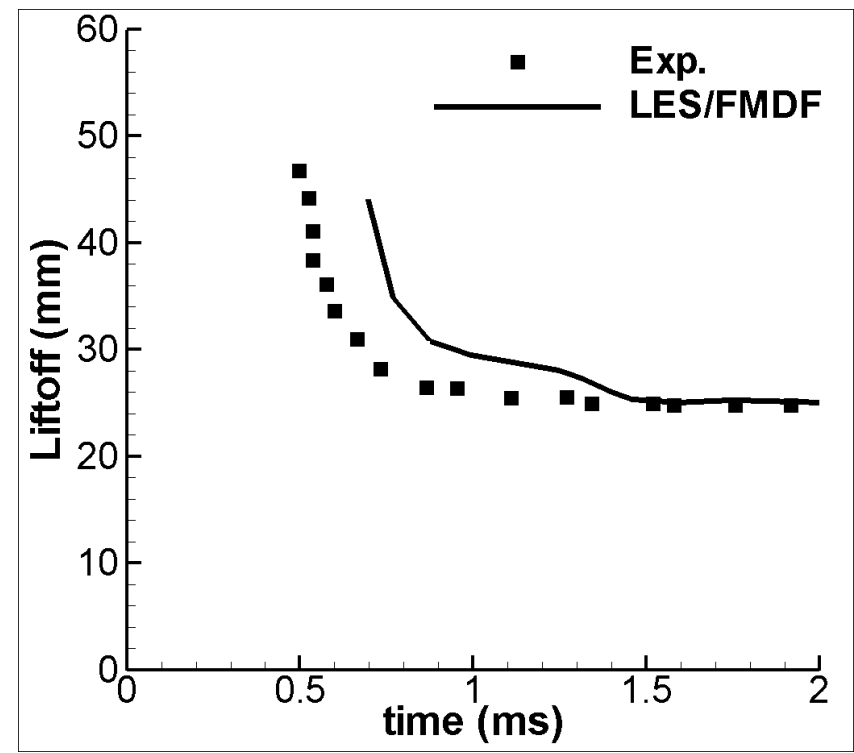

Figure 16: Time evolution of liftoff length for $\mathrm{T}=1000 \mathrm{~K}$ and $15 \% \mathrm{O}_{2}$ level.

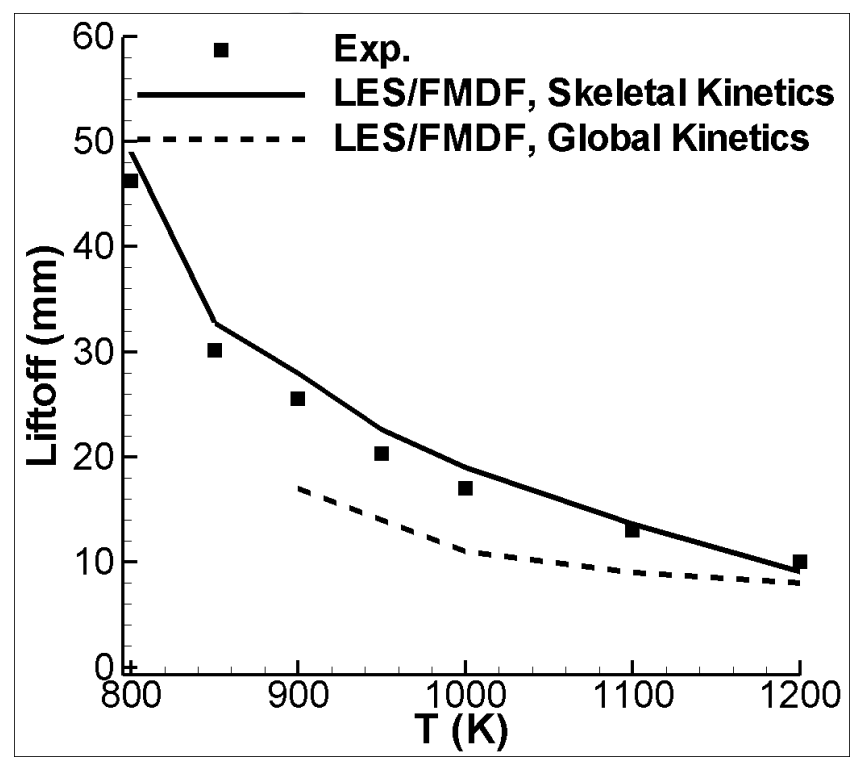

Figure 17: Liftoff length versus temperature computed by LES/FMDF/Spray model with the global and skeletal chemical kinetic mechanisms. 


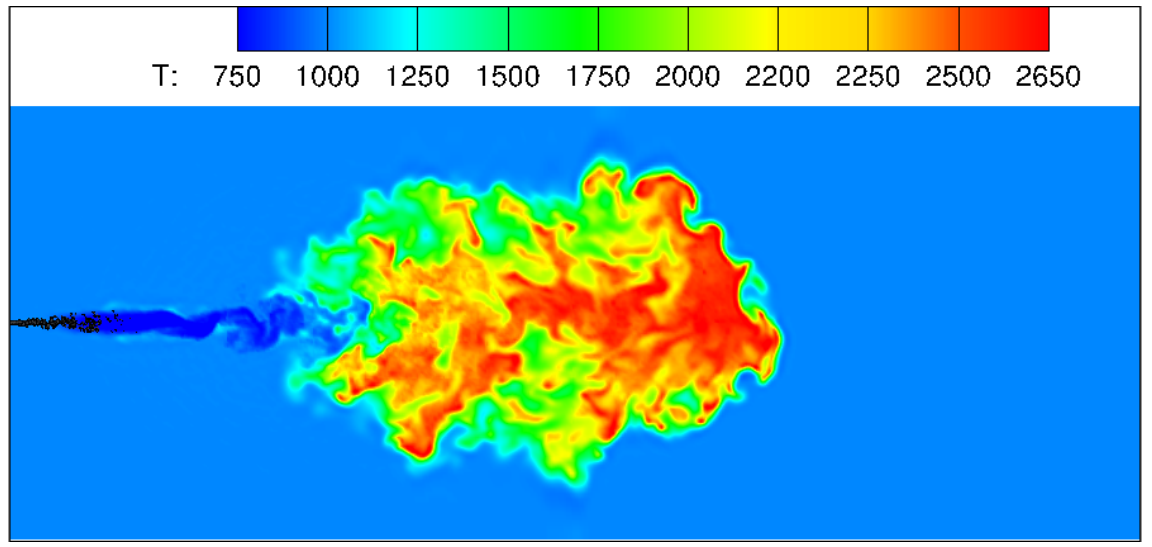

(a)

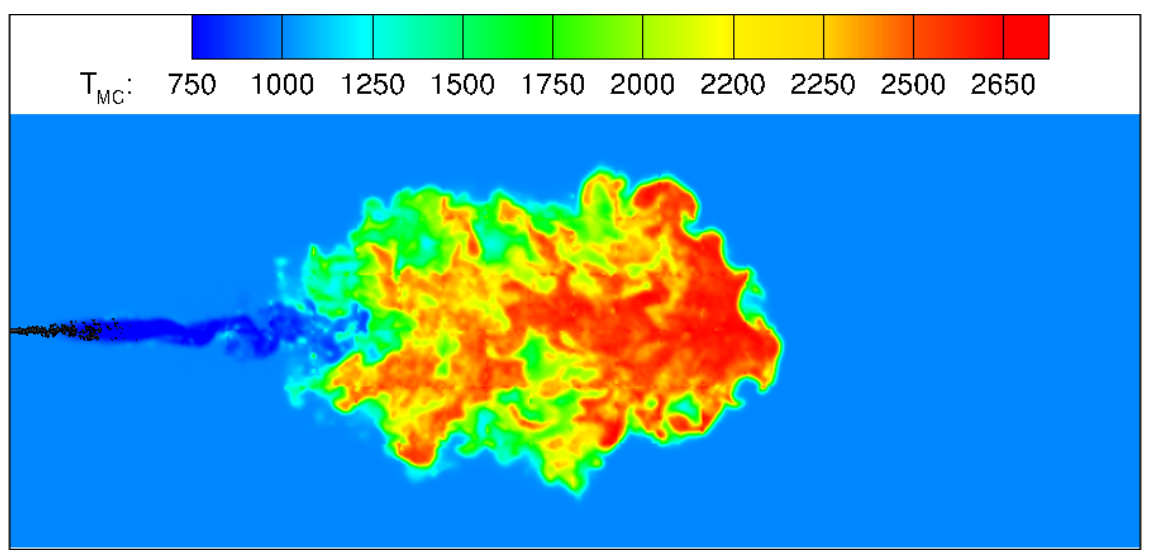

(b)

Figure 18: Gas temperature contours obtained from (a) LES-FD, and (b) FMDF-MC data, at $\mathrm{t}=2.00 \mathrm{~ms}$ for the base case. 


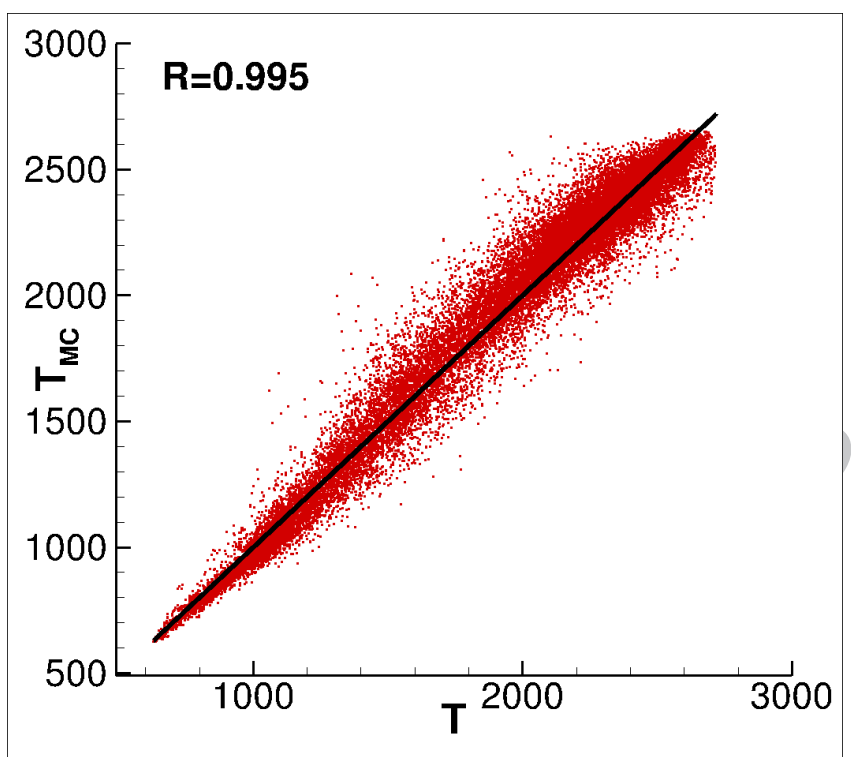

(a)

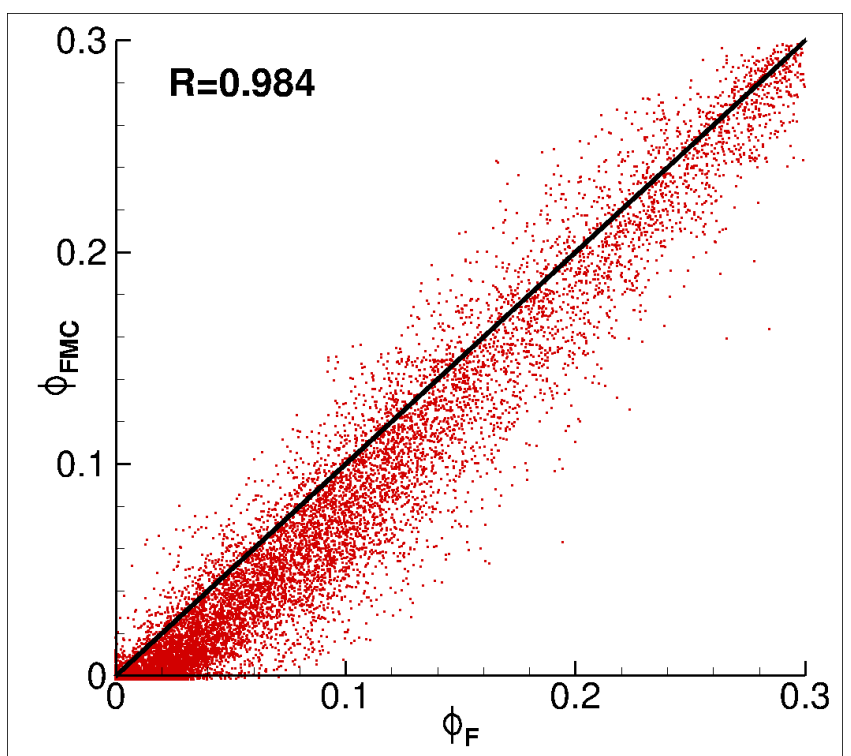

(b)

Figure 19: Scatter plots of temperature and gas fuel mass fraction for the base case. (a) temperature, (b) fuel mass fraction. 


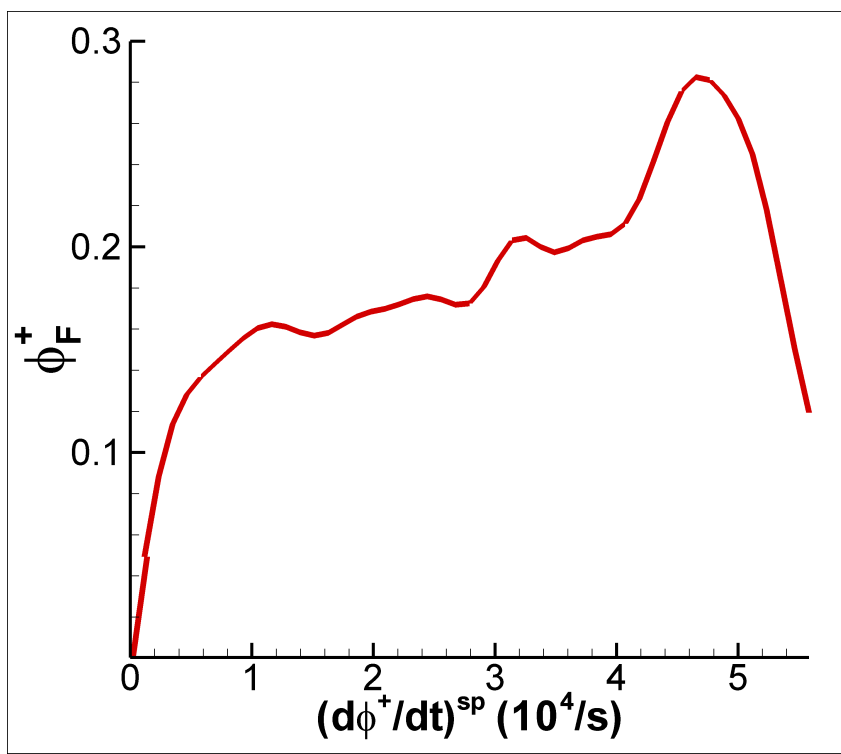

(a)

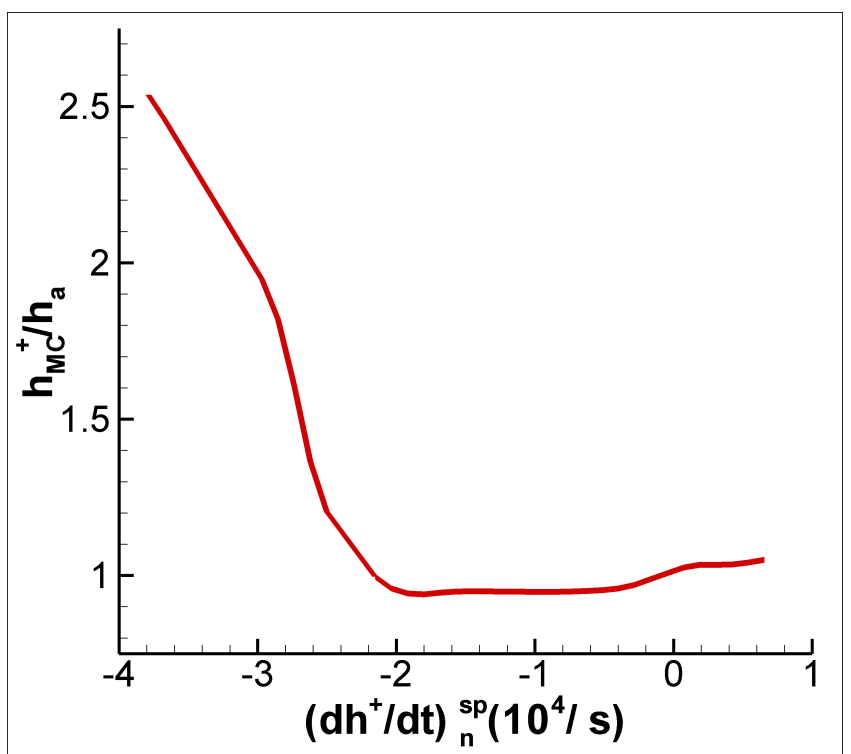

(b)

Figure 20: Mean MC particle variables conditioned on their respective spray source terms. (a) fuel vapor mass fraction, (b) enthalpy normalized with ambient enthalpy. 


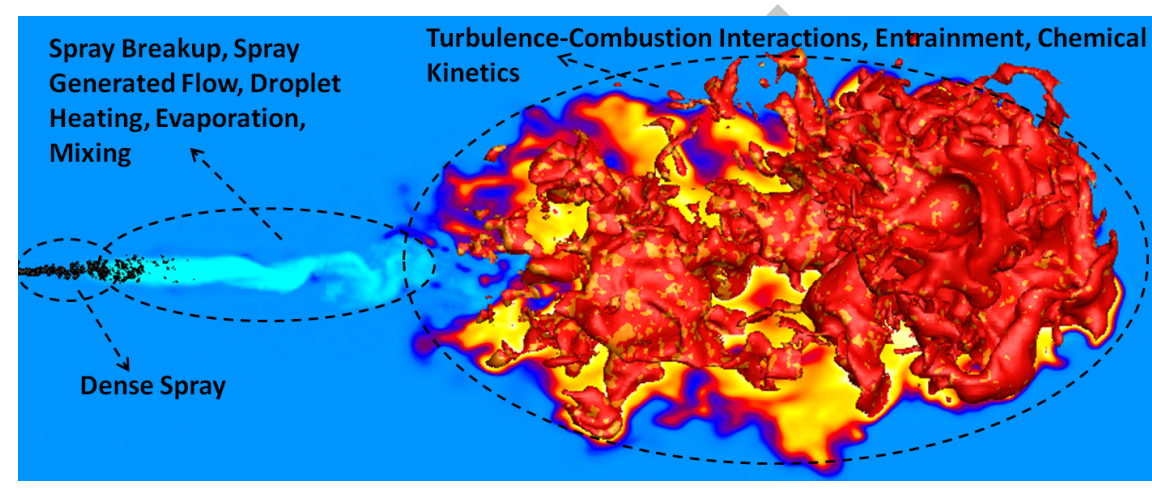

Figure 21: Various parts of the reactive high speed turbulent spray simulated by LES/FMDF model. Iso-levels of stoichiometric equivalence ratio are shown on background temperature contours. 

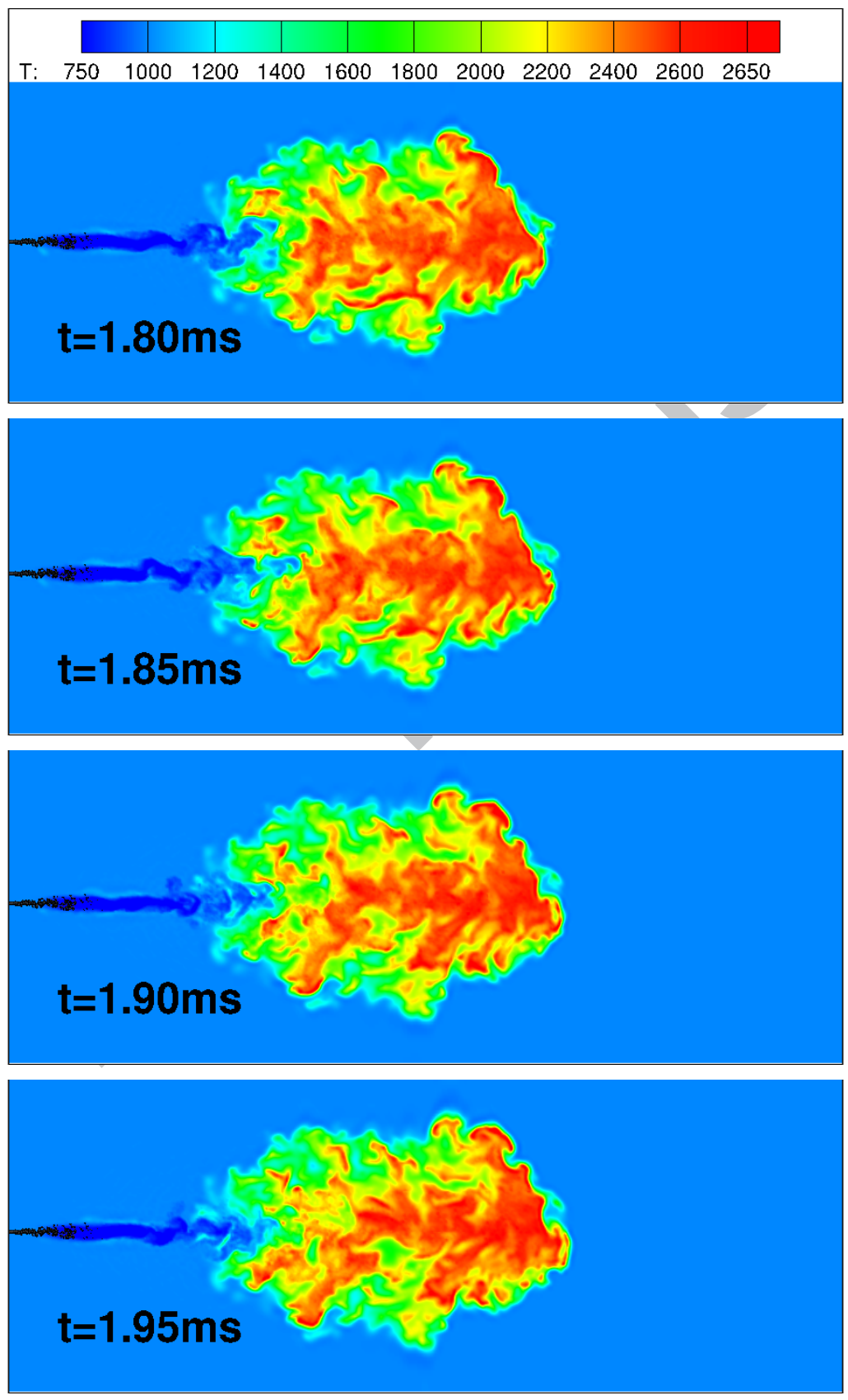

Figure 22: Contours of reacting spray temperature at different times for the base case. 


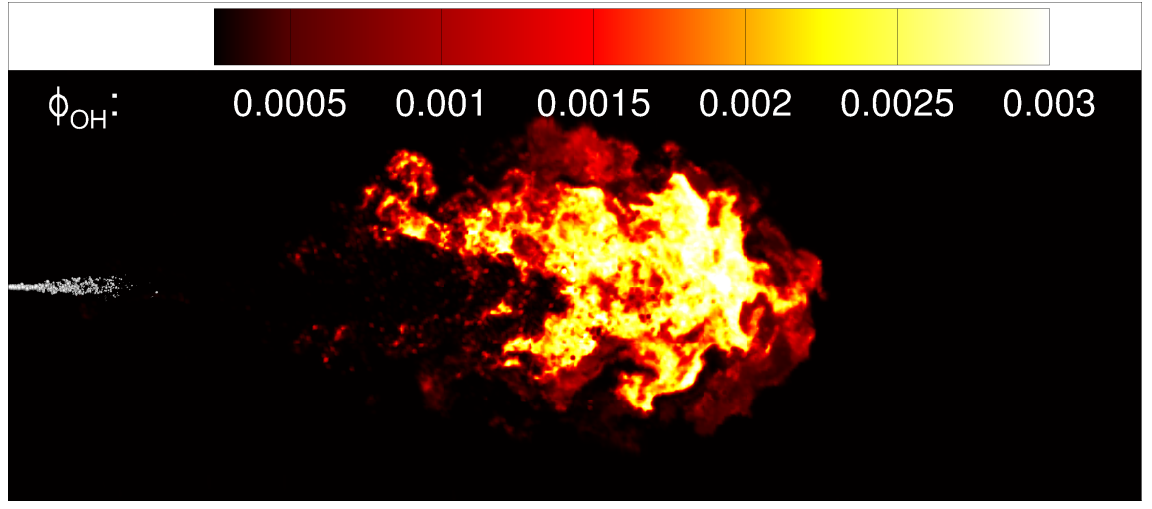

(a)

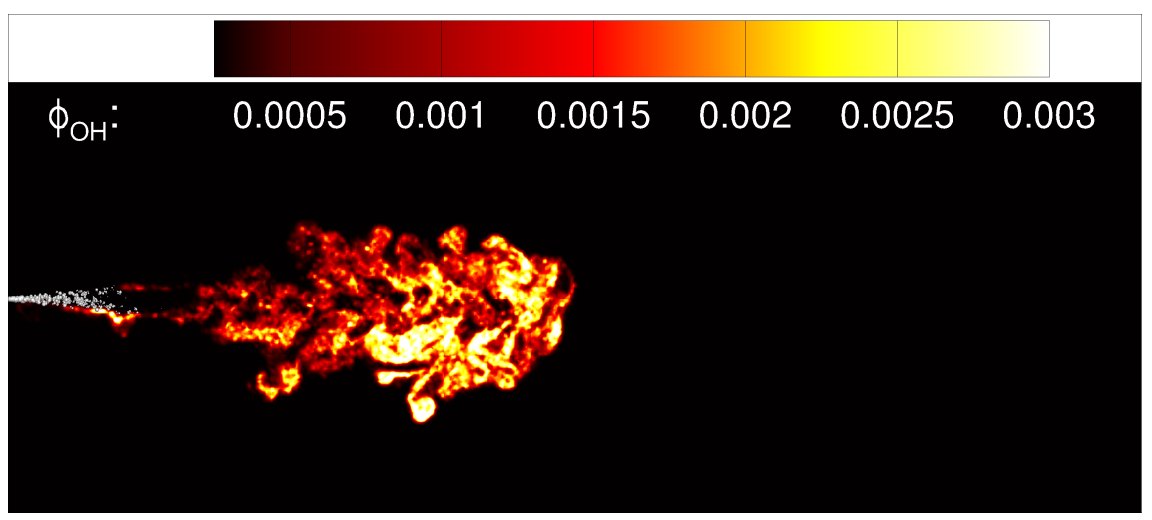

(b)

Figure 23: $\mathrm{OH}$ mass fraction contours for two different ambient gas temperatures. (a) $\mathrm{T}=850$ $\mathrm{K}$, (b) $\mathrm{T}=1200 \mathrm{~K}$. 


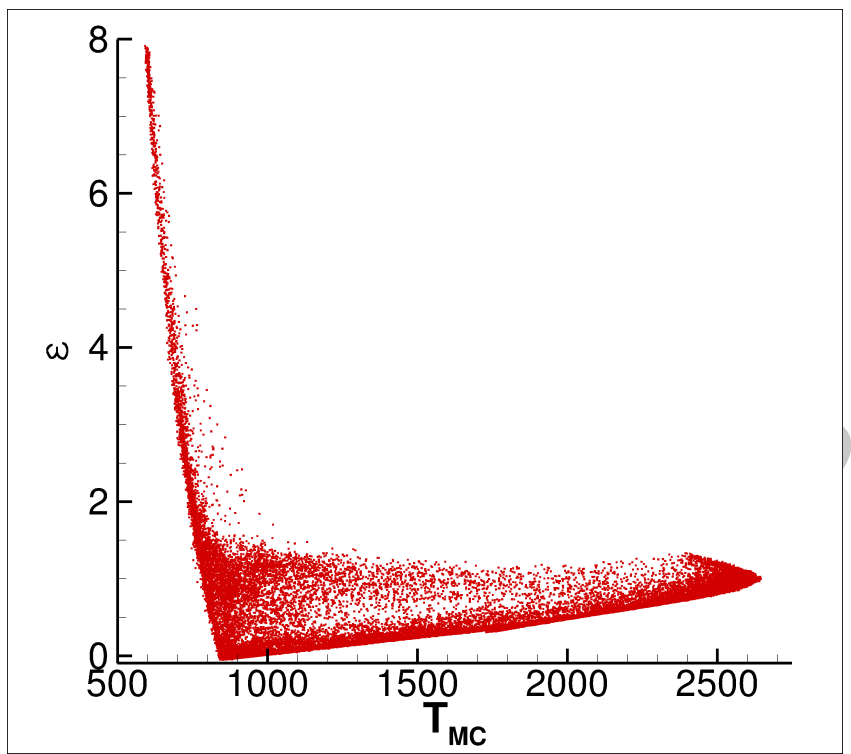

(a)

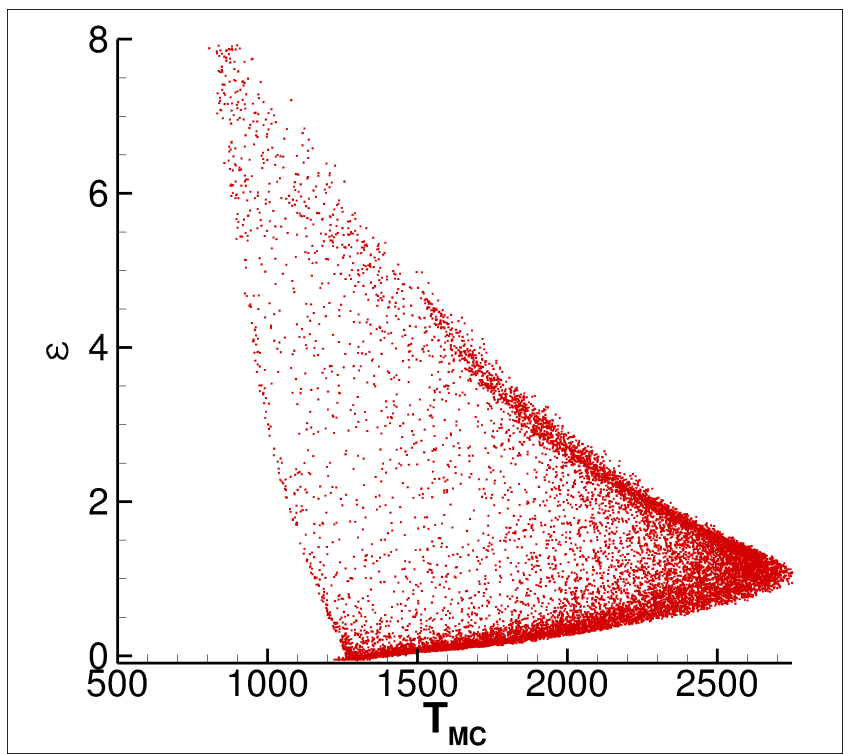

(b)

Figure 24: Maps of equivalence ratio versus temperature for two different ambient gas temperatures. (a) $\mathrm{T}=850 \mathrm{~K}$, (b) $\mathrm{T}=1200 \mathrm{~K}$. 


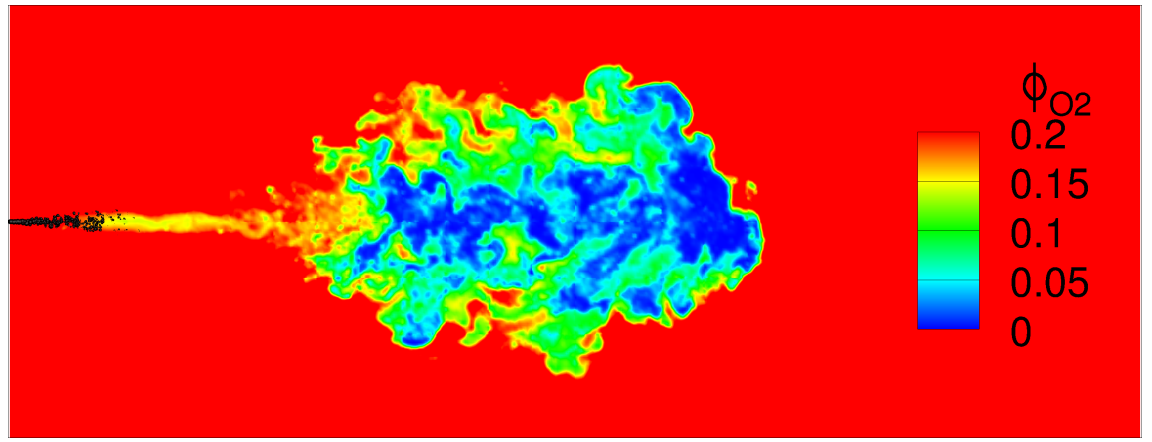

(a)

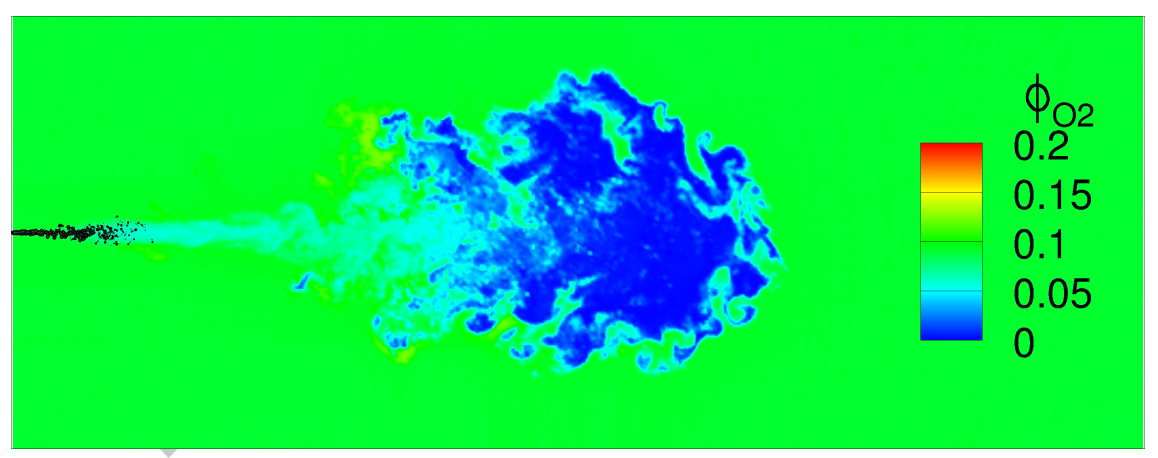

(b)

Figure 25: Comparison of normalized $\mathrm{O}_{2}$ mass fractions for sprays injected into the chamber with different initial $\mathrm{O}_{2}$ molar percentages. (a) $21 \% \mathrm{O}_{2}$, (b) $8 \% \mathrm{O}_{2}$. 


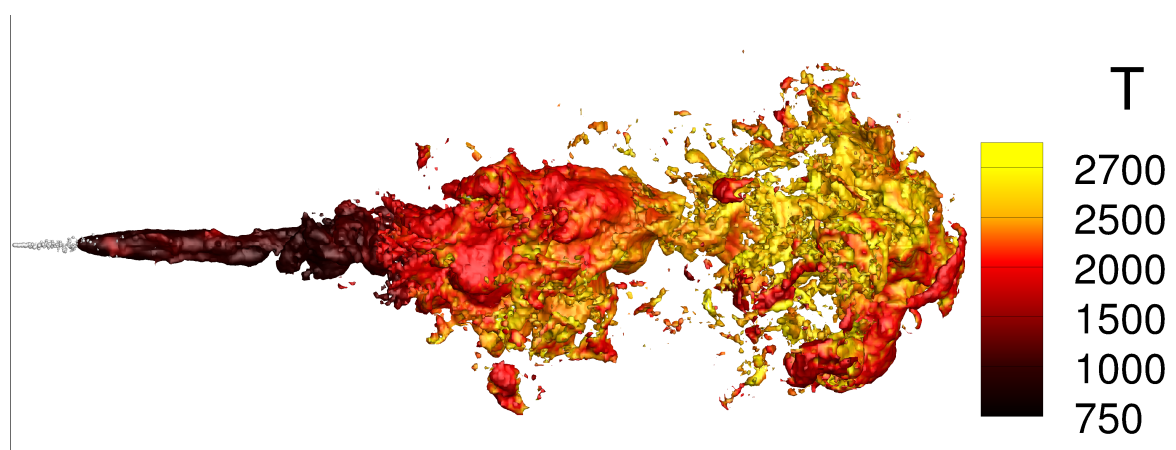

(a)
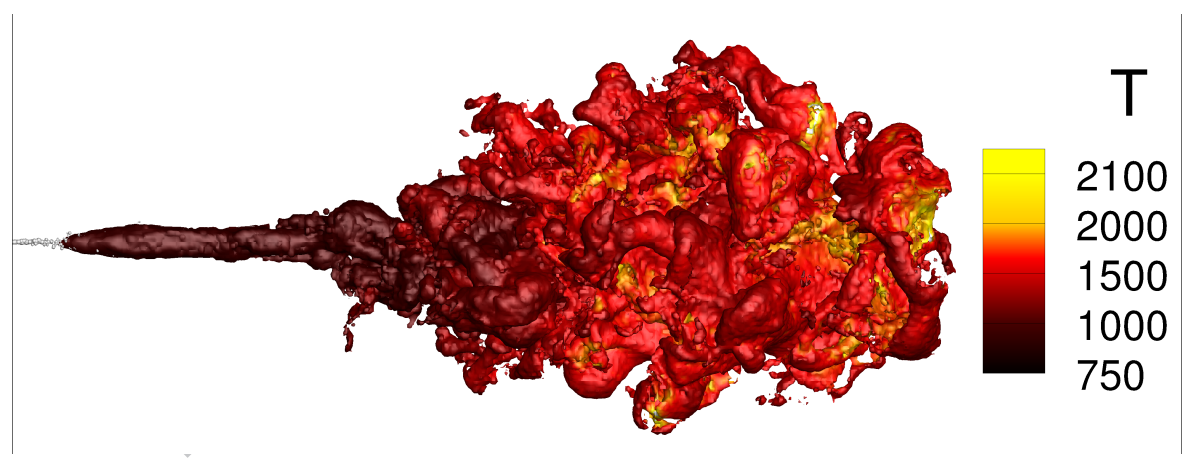

(b)

Figure 26: Iso-surfaces of stoichiometric (unity) equivalence ratio coloured by temperature contours for different oxygen concentrations at $\mathrm{t}=2.00 \mathrm{~ms}$. (a) $21 \% \mathrm{O}_{2}$, (b) $8 \% \mathrm{O}_{2}$. 


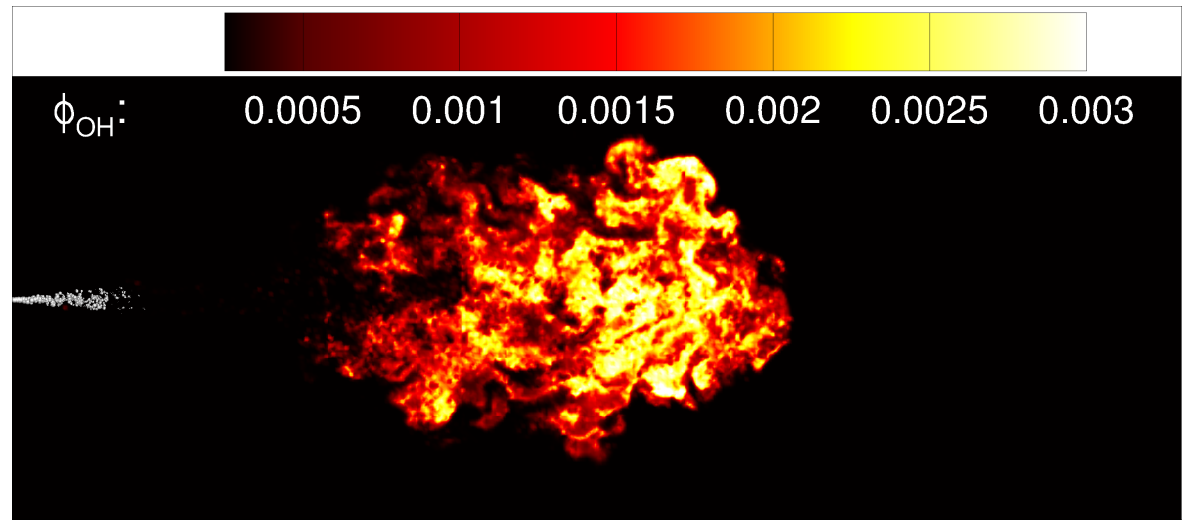

(a)

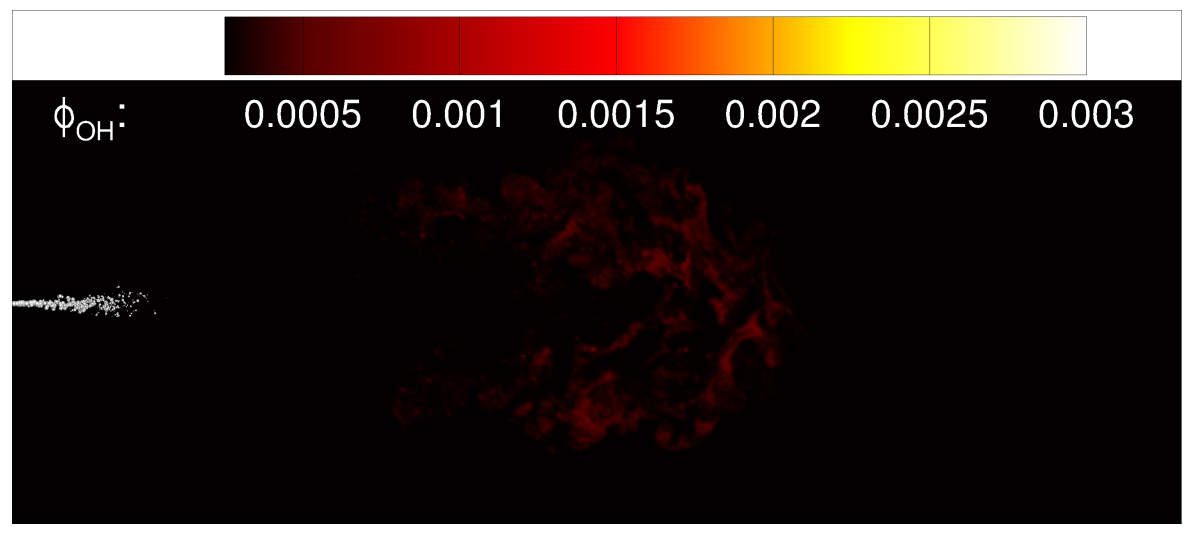

(b)

Figure 27: $\mathrm{OH}$ mass fraction contours for different initial oxygen concentrations at $\mathrm{T}=1000$ $\mathrm{K}, \rho=14.8 \mathrm{~kg} / \mathrm{m}^{3}$. (a) $21 \% \mathrm{O}_{2}$, (b) $8 \% \mathrm{O}_{2}$. 


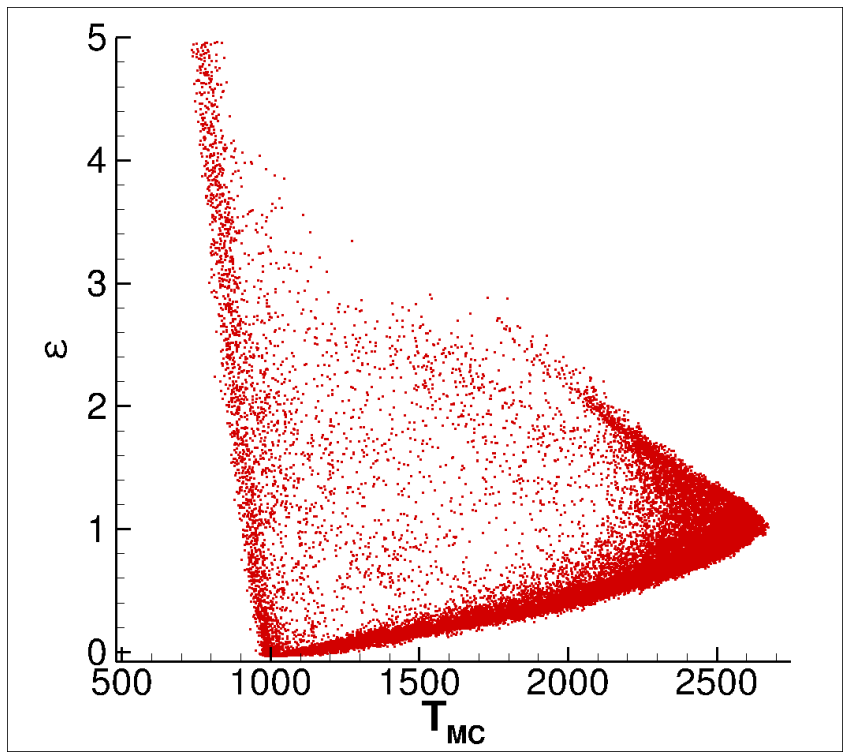

(a)

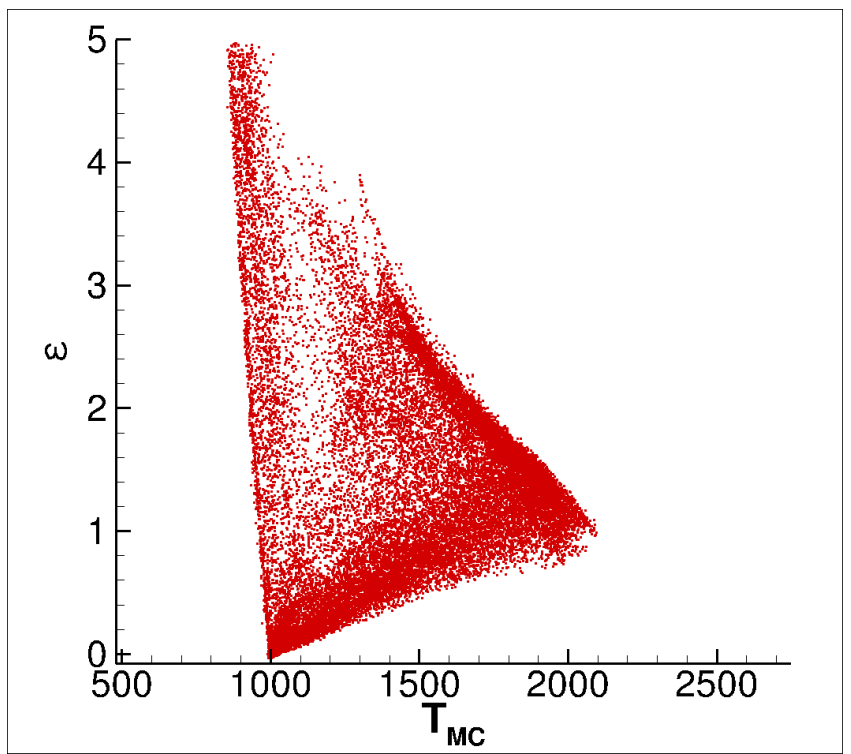

(b)

Figure 28: Maps of equivalence ratio versus temperature for different oxygen concentrations. (a) $21 \% \mathrm{O}_{2}$, (b) $8 \% \mathrm{O}_{2}$. 